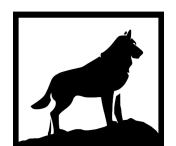

Michigan Technological

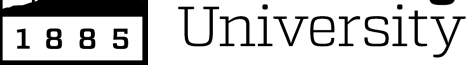

Michigan Technological University Digital Commons @ Michigan Tech

2016

INFORMATION TECHNOLOGY AND COMPUTER SCIENCE EDUCATION AT ST. PATRICK'S COLLEGE IN THE REPUBLIC OF VANUATU

Timothy R. Ward

Michigan Technological University, tward@mtu.edu

Copyright 2016 Timothy R. Ward

Recommended Citation

Ward, Timothy R., "INFORMATION TECHNOLOGY AND COMPUTER SCIENCE EDUCATION AT ST. PATRICK'S COLLEGE IN THE REPUBLIC OF VANUATU", Open Access Master's Report, Michigan

Technological University, 2016.

https://doi.org/10.37099/mtu.dc.etdr/221

Follow this and additional works at: https://digitalcommons.mtu.edu/etdr 


\title{
INFORMATION TECHNOLOGY AND COMPUTER SCIENCE EDUCATION AT ST. PATRICK'S COLLEGE IN THE REPUBLIC OF VANUATU
}

By

Timothy R. Ward

\begin{abstract}
A REPORT
Submitted in partial fulfillment of the requirements for the degree of MASTER OF SCIENCE

In Computer Science
\end{abstract}

MICHIGAN TECHNOLOGICAL UNIVERSITY

2016

(C) 2016 Timothy R. Ward 
This report has been approved in partial fulfillment of the requirements for the Degree of MASTER OF SCIENCE in Computer Science.

Department of Computer Science

Report Advisor: Dr. Linda Ott

Committee Member: Dr. Jean Mayo

Committee Member: Dr. Charles Wallace

Department Chair: Dr. Min Song 
Long pipol blong Vanuatu 


\section{Table of Contents}

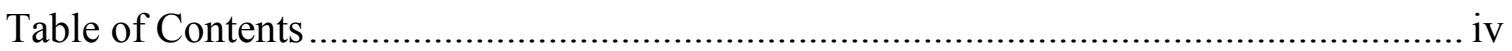

Preface

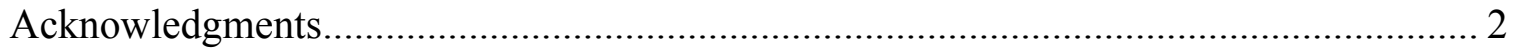

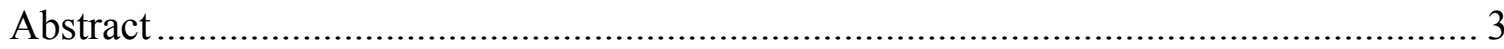

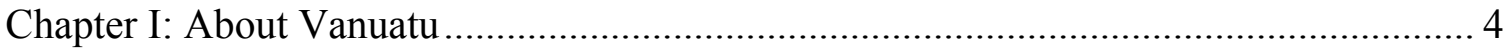

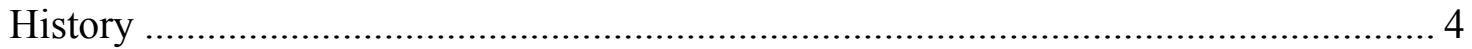

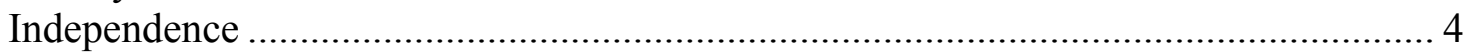

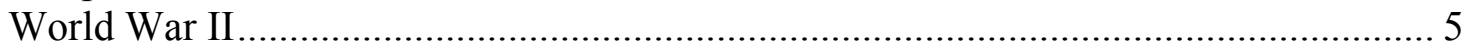

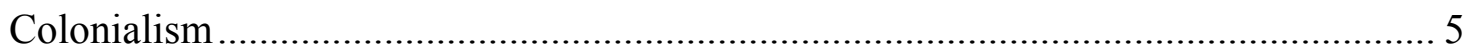

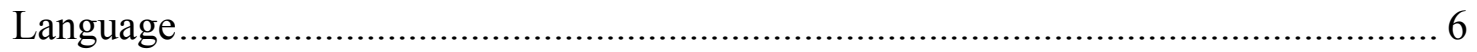

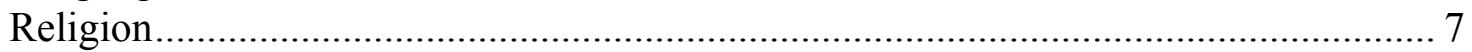

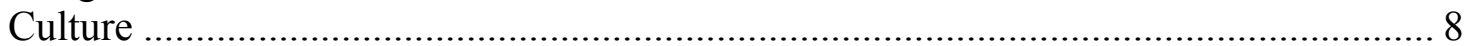

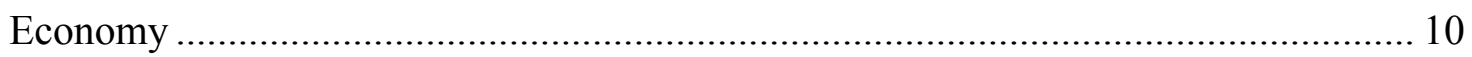

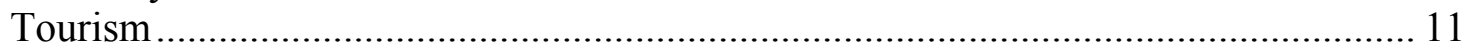

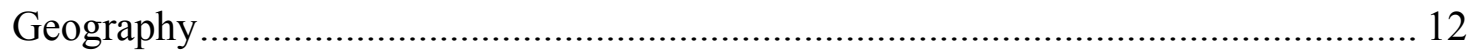

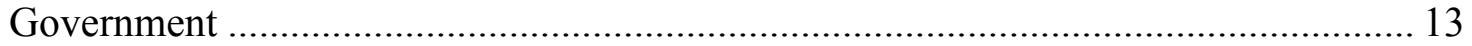

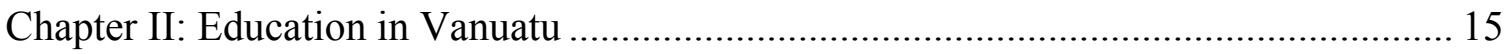

Organizational Structure ...................................................................................... 15

School Types and Student/Teacher Composition ................................................... 16

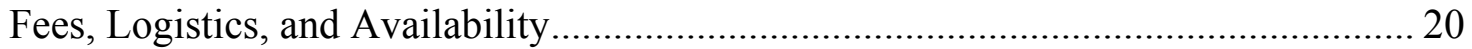

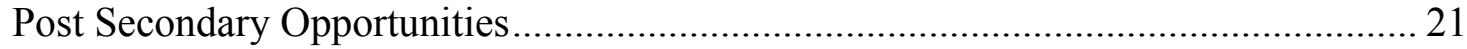

History of St. Patrick's College ................................................................................ 22

St. Patrick's College: A School of Two Thoughts...................................................... 23

History of the IT Program at St. Patrick's College ..................................................... 24

Student Socio-Economic Backgrounds and Demographics ................................... 28

Arrival of a New Peace Corps Volunteer at SPC .................................................... 31

Throughout Service: Internet, Electricity, and the Computer Lab .......................... 32

Chapter III: Curriculum Development and Improvement ......................................... 34

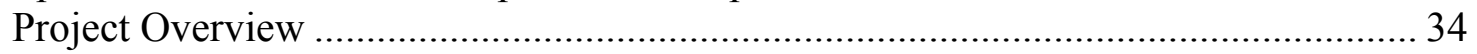

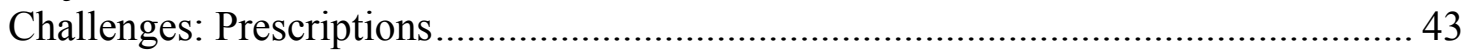

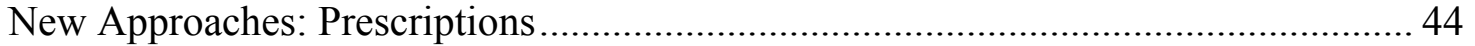

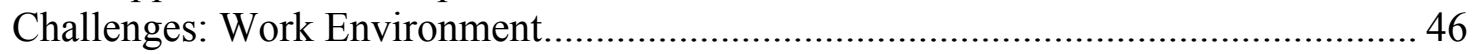

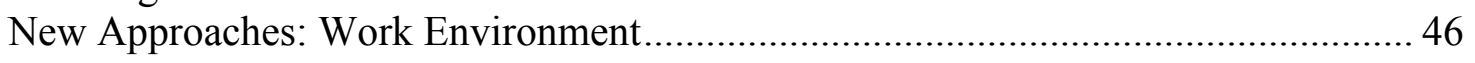

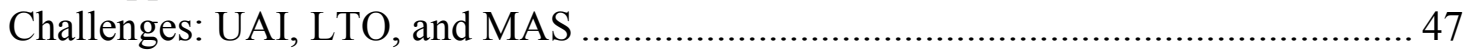

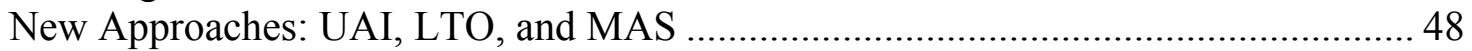

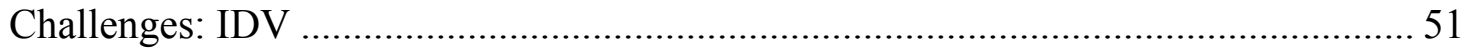




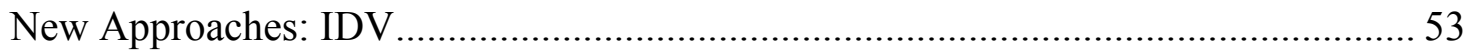

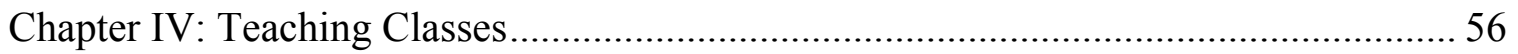

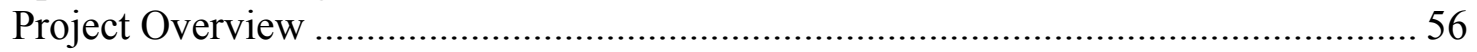

Challenges: Curriculum Choices ………………………………………………..... 57

New Approaches: Curriculum Choices ………………………………………........ 58

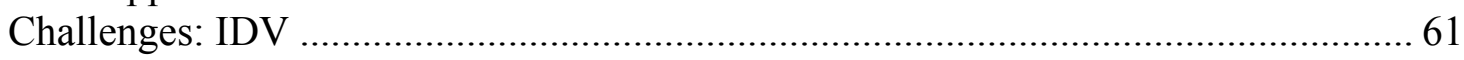

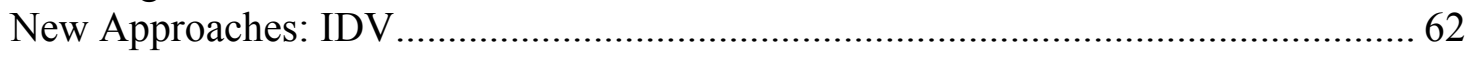

Challenges: PDI .

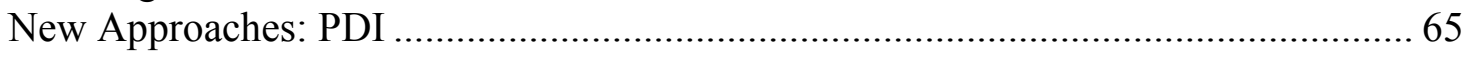

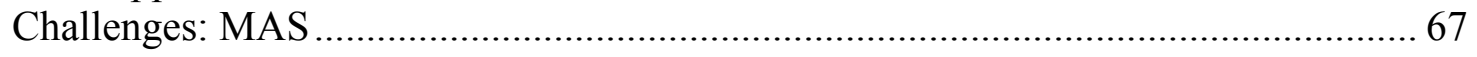

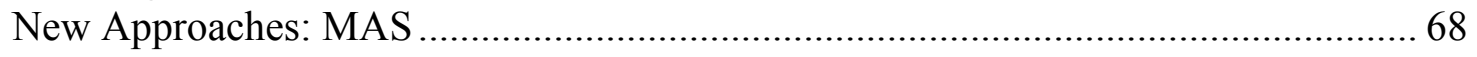

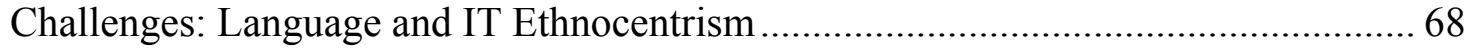

New Approaches: Language and IT Ethnocentrism .................................................. 70

Chapter V: SPC Staff Capacity Building ....................................................................... 73

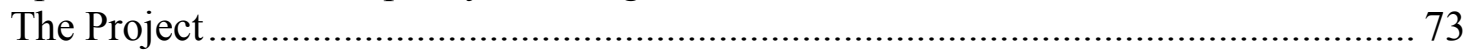

Teaching/Curriculum Crossover.......................................................................... 76

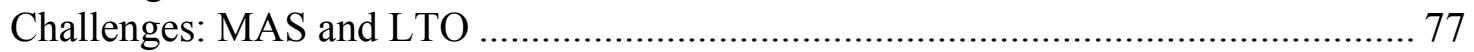

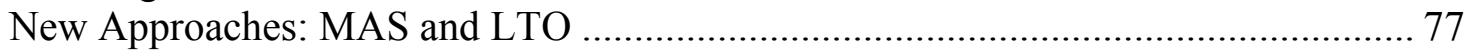

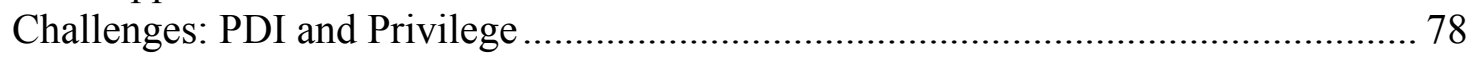

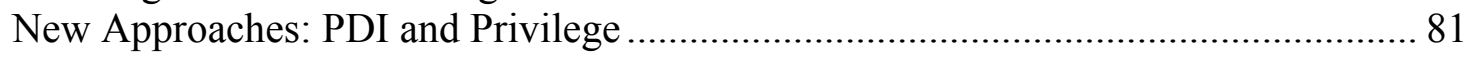

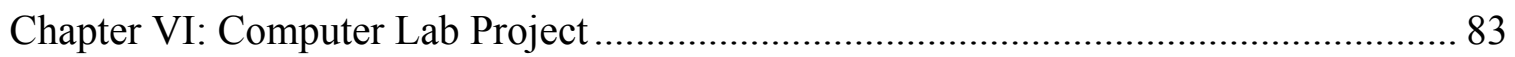

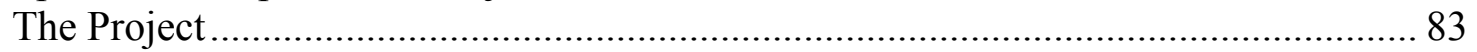

Challenges: Logistics, Electricity, Environment, Money …………………………..... 83

New Approaches: Logistics, Electricity, Environment, Money …………………….... 86

Challenges: Software and Internet ............................................................................... 89

New Approaches: Software and Internet ............................................................. 90

Chapter VII: Gender and Development Project.......................................................... 91

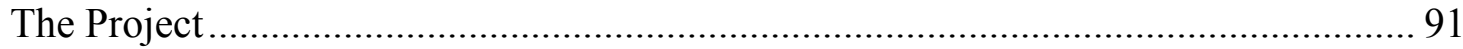

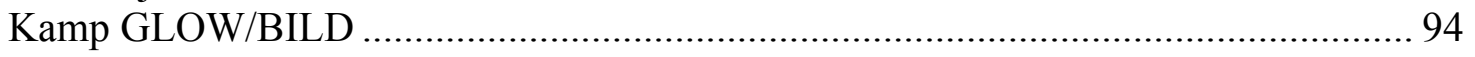

Gender Based Violence Prevention ........................................................................... 95

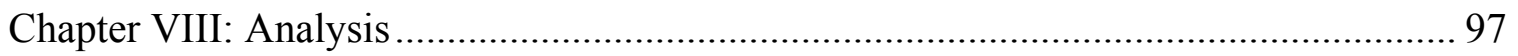

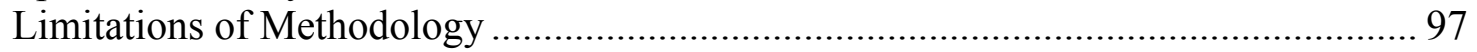

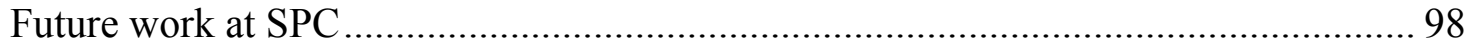

Related Work in the United States........................................................................... 99

Cultural Challenges and New Approaches ……………............................................ 100

Logistical Challenges and New Approaches ............................................................. 104

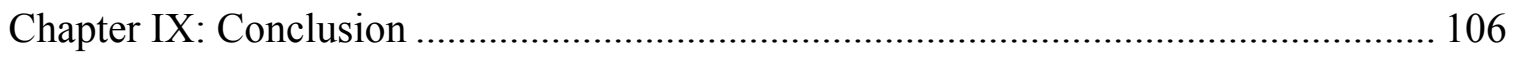

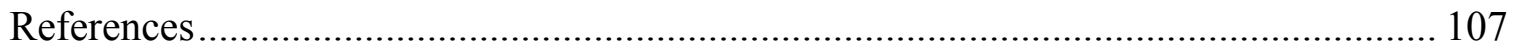

Appendix A: The History Of St. Patrick's College ………………………………….... 108 


\section{Preface}

My journey has taken me on many paths, but none so interesting and different and full of hope and life changing as that of joining the Peace Corps. I started my postsecondary education at Michigan Technological University as a Computer Science undergraduate student in the fall of 2008. By 2012 my inner drive to serve had reached a boiling point. I knew I wanted to serve a cause on this earth greater than myself. I was lying in bed in London on study abroad when I got a tweet from my university. It was a link containing information about the Peace Corps Masters International (PCMI) program. About 5 hours later, I realized I had read just about everything there was on the internet that even remotely pertained to it. You could say that there was a bit of interest on my part. Information Technology, its role in education, and its role in the developing world has always been a fascinating topic to me. The only problem was that there were no Computer Science PCMI programs. If any school could create one however, it was mine. Michigan Tech has the largest and most successful PCMI program in the country. Fast forward a bit and I deployed in January of 2014 to the Republic of Vanuatu. Having completed one year of graduate level Peace Corps prep classes, I was more excited and prepared than anyone could possibly be... until I got there. Prepared is not a word one uses lightly when working in the developing world. This, among many other things, is one of the life-changing lessons that I learned as a Computer Science PCMI student in Peace Corps Vanuatu. My primary assignment while deployed through September 2015 was to teach Information Technology and Computer Science at St. Patrick's College. This report details my story and what I learned along the way. 


\section{Acknowledgments}

Mom, for everything and so much more.

The Volunteers and Staff of Peace Corps Vanuatu, for giving me strength.

Hambert and Nellie Toa, for being the best host family a volunteer could hope for.

Dr. Linda Ott, for conversations, laughs, and advice well received.

Dr. Blair Orr, for a vision sparked into a flame of spirit guided by wisdom.

Keith Honda, for illuminating insights and being greater fools together.

Dr. Kari Henquinet, for tireless patience, grace, and poise, in seeing it through.

Dr. Jean Mayo, for working where I could not, with an open mind, from the beginning.

Dr. Charles Wallace, for putting up with me and for understanding. 


\begin{abstract}
This report examines the role of an Information Technology and Computer Science Education Volunteer with the United States Peace Corps in the Republic of Vanuatu at St. Patrick's College. Various methods of developing curriculum, training teachers, and teaching classes were used and the results are analyzed. Primarily cultural and logistical challenges and their associated newly discovered approaches are discussed. Some approaches were found to improve Information Technology and Computer Science Education at St. Patrick's College.
\end{abstract}




\section{Chapter I: About Vanuatu}

\section{History}

The history of a nation as culturally rich as Vanuatu cannot be explained in a few short paragraphs, but if one were to try, they would begin with Kastom. Kastom is a word in the local language ${ }^{1}$ that refers to all of the culturally held beliefs, practices, and history of Vanuatu. It includes aspects of religion, family, business, trade, colonialism, geography, black magic, tradition, and language. The most approximate English translation might be the word "culture" but it is more commonly used to specifically refer to cultural practices like ceremonies, traditions, and social structures.

Vanuatu was first settled by people speaking Austronesian languages around 3300 years ago using canoes to travel to the volcanically formed islands. In 1606 the Portuguese explorer Pedro Fernandes de Queirós was the first European to discover what James Cook would later (in 1774) name the New Hebrides. However, Pedro was mistaken and thought he had discovered the beginnings of Terra Australia (the Australian Continent) and thus named it La Austrialia del Espiritu Santo, of which Espiritu Santo remains the name of that island to this day [1].

\section{Independence}

The primary influence over the last few centuries on the country has been in two forms. First is that of colonialism. Second is religion, specifically Christian missionaries. The islands themselves were occupied by the French and British jointly as the New

\footnotetext{
${ }^{1}$ Local language is a slightly problematic term here. In Vanuatu this actually refers to one of hundreds of actually local languages. Here however, this refers to Bislama.
} 
Hebrides up until the year 1980, when Walter Lini, the "Father of Independence" led the people to adopt a constitution and become the independent island nation we know today as Vanuatu. The very name Vanuatu means freedom and comes from the root words "vanua" and "tu" in several Austronesian languages, meaning "land" and "stand" respectively [2]. Vanuatu's pre-independence history is very complicated and less relevant to the topics in this report, so I will only cover Vanuatu's history from the perspective of the major themes that drive Vanuatu's post independence identity.

\section{World War II}

The second world war had a profound impact on Vanuatu. There are only two cities in the country, Port Vila the capital and Luganville on the largest island, Espiritu Santo. Port Vila is largely the result of urban sprawl and a city grown purely from tourism and trading interests. Luganville however is actually the remnants of a former US military base used in World War II as a staging area to attack the Japanese as well as a rest and relaxation base for armed forces personnel on leave. This among other WWII influences, was the first nation-wide exposure to Americans and militaries in general for the people of Vanuatu. The USS Calvin Coolidge remains a very popular Scuba Diving attraction after the ship was sunk because the US military forgot that they had covered the largest channel with mines.

\section{Colonialism}

Colonialism left its mark on the nation and its people. One could even argue that Vanuatu is more defined by colonialism than it is defined by pre-colonialism. The economy began a radical transformation from subsistence farming to agrarian market 
based, one which is still underway today. The social structures, particularly with regard to gender roles and how white people are perceived were radically altered. Healthcare moved from traditional Kastom medicine to incorporate more western ideals as well as problems like nicotine and alcohol addiction that still plague the nation today. A new music formed in to what is now known as "stringband" which is a combination of string instruments, reggae, and electronic synthesizers. Kastom changed and in many places died completely. Politics and government were adapted to more western ideals so as to fetch foreign aid dollars. Food and cuisine began to incorporate the more convenient but usually less healthy foods of other nations like rice, candy, and pop. But the two largest factors of colonialism that radically altered the people and culture of Vanuatu the most (primarily because they affect so many other things) were language and religion.

\section{Language}

Vanuatu is an extremely language-diverse place, with over 113 local languages actively spoken throughout the 82 islands today [3]. Because of this, there is much work in Vanuatu around language preservation, particularly in the form of biblical translation. The language of business/education is primarily English and secondarily French, although the French would have you believe otherwise. This is a controversial political issue in Vanuatu. The language of everyday use and communication both between natives and non-natives, is called Bislama. Bislama is a pidgin language derived primarily from English. The local populations learned to communicate in rudimentary English in order to facilitate trade and over time Bislama was formed. Bislama has now reached the point (particularly in urban areas) where children learn it as a first language and, mixed with other languages and grammars, it is even progressing into a stable 
creole. In addition to radical language changes, colonialism brought with it one other factor that affected many aspects of Vanuatu and its people. Religion.

\section{Religion}

Religion has played a large role in Vanuatu's history and shaped much of what the country has become today. About $83 \%$ of the country self-reports being Christian, with many denominations [4]. So strong is this influence, that Bislama has no word for denominations, which are simply known as various "religions" there. The primary source

of this influence came (and still does come) from missionary work. Usually in the form of international aid, missionaries have done work on each and every island of Vanuatu. The school that I worked at (being the primary focus of this report) was, for instance, an England-based Anglican owned, operated, financed, and styled private boarding senior secondary school. Religion and missionary work itself is a sensitive topic among academics familiar with Vanuatu as many of the influences left behind resulted in the destruction of local culture, language, beliefs, and social structures. For instance, under old Kastom laws, women could hold chiefly rank in the province of Penama. Such was not the case following Christianity's hold. In addition to Christianity there were many (and a few still exist) cargo cults that cropped up as a result of America's influence during WWII. These cults include that of John Frum and the Prince Phillip Movement. Language and religion play a pivotal role in every aspect of Vanuatu and help define much of the culture of Vanuatu to this day. 


\section{Culture}

Talking about culture is a difficult thing to do, mostly because it is such an amorphous concept. Using Hofstede's Cultural Dimensions (HCDs) helps to guide the discussion for those who have not lived in and integrated into a specific culture. They will be used throughout this report to guide the discussion in a more structured way where possible. The HCDs are defined below using the definitions from www.geerthofstede.com and using context-sensitive examples of my creation [5].

\section{- Power Distance (PDI)}

$\circ$ The extent to which the less powerful members of society accept that power is distributed unequally.

- Ex: How comfortable you are as a junior staff member talking to a principal? Comfortable indicates low PDI, uncomfortable indicates high PDI. Inversely, how comfortable is a principal receiving such feedback?

\section{- Individualism (IDV)}

o Individualism: people only look after themselves and their immediate family.

- Ex: The computer in the principal's office is only for him/her and the school truck is only for school business and only driven by the driver.

o Collectivism: people belong to in-groups (families, organizations, etc.) who look after them in exchange for loyalty.

- Ex: The computer in the principal's office is for everyone to use, the school truck is for anyone who has need of it, and anyone can drive it.

- Masculinity (MAS)

o Masculinity: the dominant values in society are achievement and success.

- Ex: You might introduce yourself at a meeting with your first name, where you work, what you are interested in, and what your goal is at this meeting.

o Femininity: the dominant values in society are caring for others and quality of life.

- Ex: You might introduce yourself at a meeting with your family name, giving thanks for the meeting organizers/venue/God/everyone, where you come from, your religion, family make-up, and how you feel about the meeting.

- Uncertainty Avoidance (UAI)

o The extent to which people feel threatened by uncertainty and ambiguity and try to avoid such situations.

- Ex: As a teacher, you might make a lesson plan with meticulous detail if you have a high UAI. With a low UAI, you might just go with the flow and do what feels right in the moment.

\section{- Long Term Orientation (LTO)}


○ The extent to which people show a pragmatic or future-oriented perspective rather than a past/present-oriented or short-term point of view.

- Ex: As a community leader with a low LTO (high STO) you might be more concerned with your day to day social status, which is maintained by

drinking at bars and having fun. With a high LTO (low STO) however, you might be more wanting to avoid drinking with your friends in order to get some work done and get ahead.

\section{- Indulgence (IND)}

o The extent to which people try to control their desires and impulses.

Relatively weak control is called "Indulgence" and relatively strong control is called "Restraint".

- Ex: A high IND (low RES) individual might be in need of purchasing something and does so with 2-day shipping. A low IND (high RES) individual might simply plan to pick up said item the next time they are in the city where it can be bought, borrow it from someone else, or buy it in 6 months if they still need it then.

Throughout the discussion in this report, these HCDs will be discussed as

probable causal factors for why certain methods failed, were only marginally successful, or were very successful. Knowing the index values for Vanuatu relative to the Western World (specifically America in this case) is therefore very important. Unfortunately, HCD data is not available for Vanuatu, so the next best (most similar culture) numbers come from Fiji, which are incomplete. The LTO and IND indexes are not available. The grey bars indicate the values for me personally. These personal scores come from the 
same website using their online assessment tool. [5]

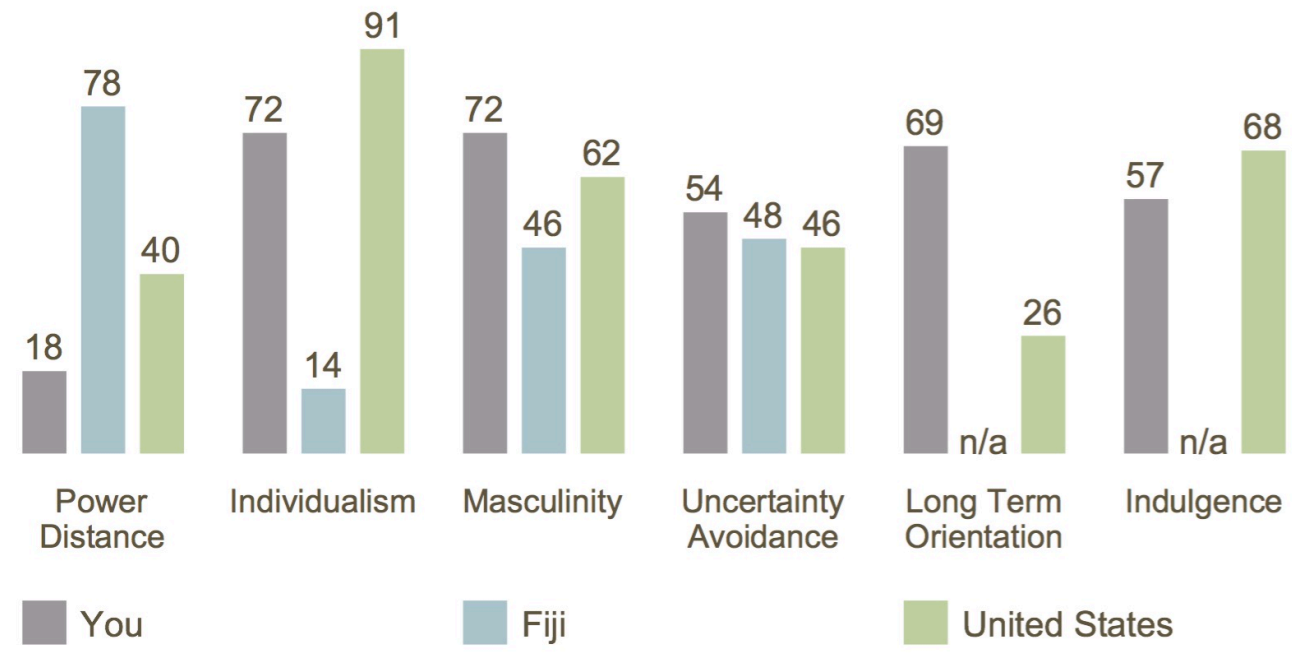

Figure 1: HCD Values for the author, Fiji, and the United States. Source: http://geert-hofstede.com/ The actual values for Vanuatu, using an entirely unscientific approximation from my own experiences, are probably a bit different. For example, I would think MAS and UAI are much lower than Fiji. I would also hazard a guess that both Vanuatu and Fiji's LTO and IND values are very low. This makes sense given that Fiji is a more developed and more technologically advanced nation with a higher population and countries typically follow this trend as they become a more developed nation. Typical developednation values are mostly polar opposite from Vanuatu and Fiji. As one might imagine, this makes for a difficult environment to accomplish typical developed-nation-driven goals, like CS/IT education. Even more difficult is the typical needs of the modern economics world, like business and finance.

\section{Economy}

The modern day economy of Vanuatu is largely based on a few things. First and foremost is tourism, but that is covered in the following section. Second is agriculture and 
third is financial services. Agriculture makes sense given that the majority of the country's people are subsistence farmers. Main agricultural exports include copra, coconut oil, fish, cattle, cacao beans, kava, and Tanna ${ }^{2}$ coffee. Vanuatu's imports largely consist of processed foods like rice and canned products as well as technology from the western world, primarily via China which has an enormous hold over the country economically as nearly all major suppliers of goods are sourced from and/or owned by Chinese nationals. The financial services industry attracts some shady characters, primarily due to the services offered. Vanuatu does not collect any taxes, does not participate in the international banking system, does not reveal information about accounts (although this is slowly changing) and largely does not regulate their internal finance industry. As a result, there are accounting offices throughout the capital that largely service money that was earned/stolen outside of Vanuatu and will never be used in Vanuatu. Vanuatu is also considered a "Flag of Convenience" country for many shipping and commercial fishing operations given the lack of regulations, taxes, and labor laws. These same benefits are also useful to the (therefore extremely inexpensive) tourism industry.

\section{Tourism}

Vanuatu also has a thriving tourism market located mostly in the capital of Port Vila and the only other city, Luganville. Tourists only see other areas of the country as functions of ecotourism or cultural excursions. This market thrives because the islands are beautiful, warm, rich in culture, and conveniently inexpensive for holidays. They are also located very conveniently for Australia and New Zealand vacationers. The

\footnotetext{
${ }^{2}$ Tanna is an island in the southern province of Vanuatu, as explained in the geography section.
} 
combination of long held colonial norms and tourism influences in the two cities has created a drastically different history and culture as compared to the rest of the country. This effect is exaggerated even more so than compared to other capital-centric developing nations because Vanuatu is made up of many islands, which are difficult and expensive to navigate between. For instance, there are many people in Vanuatu that have never seen more than 2 or 3 of its 65 inhabited islands.

\section{Geography}

The geography of Vanuatu is best described as a Y-shaped archipelago. The islands are volcanically formed and very tropical with beaches everywhere. Natural hazards include cyclones, earthquakes, tsunamis, and volcanoes. Following independence, the country was divided into 6 provinces, each being made up of several islands. They are Shefa, which is home to the capital Port Vila, Sanma, which is home to

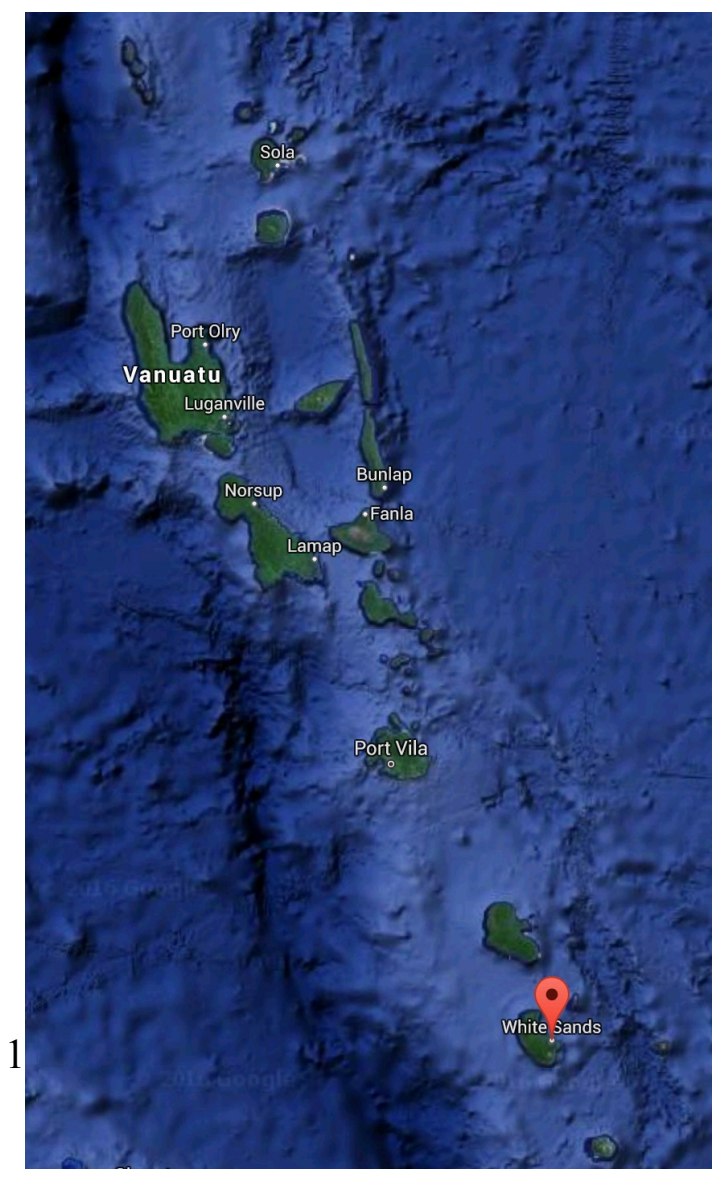


the only other city Luganville, and Malampa, Penama, Tafea, and Torba. The province names are derivations of the first few letters of each island. For instance, Penama is formed from Pentecost, Ambae, and Maewo [6].

\section{Government}

The government of Vanuatu follows its geography in the sense that each province has its own provincial government of elected/appointed ministers, officers, and departments over somewhat similar islands that also happen to be close together. This makes sense given the inability for the majority of the Ni-Vanuatu ${ }^{3}$ people to move easily throughout the country. The federal government is set up in a very similar way to most post-colonial nations of the former British Empire. The key points to note are that Vanuatu has a Council of Chiefs, a President who is largely ceremonial, a Prime Minister who serves as the head of state, a constitution, and a judicial system [7]. The constitution very much empowers chiefs in Vanuatu, in fact it could be reasonably said that paramount chiefs wield far greater power than many MPs. In addition, the council of chiefs is the paramount advising authority on all matters of Kastom, culture, language, and history. This council is made up of the most highly ranked chiefs from around the country. Depending on local Kastom, these chiefs and the lower chiefs from each area descending all the way down to the village-of-100 level are elected or inherited. Chiefly structure and how it relates to local Kastom is very complicated and varied throughout the country. Both Chiefly and Parliamentary variants of politics in Vanuatu (like most developing nations) are steeped in widespread corruption [8]. In addition, the economy

\footnotetext{
${ }^{3} \mathrm{Ni}$-Vanuatu is the correct form to refer to the people of Vanuatu as a whole, and translates literally from some of the indigenous local languages as "of Vanuatu"
} 
and politics of Vanuatu are very susceptible to money related influences seeing as an enormous amount of the economy is sourced in foreign aid. The primary day-to-day functions of the government are carried out by the Ministries and Departments, the federal heads of which are appointed at the sole discretion of the sitting Prime Minister. There are no controls over whether these individuals are qualified and this also leads to problems in a collectivist society, where using power and influence to help those of your social group is a normal, accepted, and moral form of returning loyalty. One of the largest and most important of these departments and ministries, is of course, the Ministry of Education. 


\section{Chapter II: Education in Vanuatu}

\section{Organizational Structure}

The Ministry of Education (MOE) is the primary governing authority over

educational standards, implementation, and operations of schools throughout Vanuatu. There are provincial offices of this ministry as well as the national office. Most of the work that the Ministry of Education does falls under one of two categories: curriculum development/advising and operational management/policies.

The Teaching Service is an agency that places teachers throughout the country. This agency is very powerful given that teachers in Vanuatu transfer as often as every two years. Both transfers and initial appointments are often fraught with political complications and motivations. In addition, where teachers wish to work is often not a considered factor, which results in sustainability problems both within schools and even within teachers themselves. This is because after years and years of postings at schools that teachers don't want to work at, many simply stop teaching and go back to subsistence farming with their families.

Other agencies and groups like NGOs, religious organizations, and curriculum authorities also have a significant role to play in the structure and inner workings of Vanuatu's education system. For instance, secondary schools are often tied to religious and other organizations for additional funding as well as academic authorities that provide them with a prescription that (hopefully) allows for students to have postsecondary education opportunities. This was precisely the case where I served at St. Patrick's College. 


\section{School Types and Student/Teacher Composition}

There are (for the most part) two types of schools in Vanuatu: primary and secondary, however there are many variations on this theme. Kindergarten has no curriculum, being mostly just a method to introduce kids to the school environment for the first time. It is available at some primary schools but not most. It is sometimes the only grade offered in a community due to the inability for such young children to travel long and often dangerous trails to/from school. Grades 7 and 8 are considered “extension” grades and are often attached to primary schools. This allows for more access to these grades at usually lower fees, but also usually lowers the education quality for these years.

If grades 9 and 10 are also offered, then this becomes a fully qualified junior secondary school, usually refereed to sans the "junior" qualifier. The most important difference between a full junior secondary school and a primary school with extension grades is that a junior secondary school operates as two distinct schools. The primary and secondary halves have the same name and are at the same location but operate distinctly. Each has their own headmaster/headmistress for the primary and principal for the secondary, as well as their own budgets, meetings, etc.

Schools with years ${ }^{4} 11-13$ are considered senior secondary schools. Such schools rarely offer year 11 without 12 , but often do teach 11 and 12 without 13 . This creates a 2year stepping system, which follows naturally because the examinable years are $8,10,12$,

\footnotetext{
${ }^{4}$ Note that 'grades' and 'years' are synonymous and used interchangeably.
} 
and 13. A 'college' usually refers to a school that only offers grades 7-12 and possibly 13. In 2015 , only 7 colleges offering schooling through year 13 existed in Vanuatu.

Outside of the K-13 system are Rural Training Centers (RTCs) which are a form of trade school designed for secondary school aged students that were not able to complete or enroll in secondary education for whatever reason. These institutions are usually run by outside NGOs and religious groups. For instance, St. Patrick's College (SPC) has a sister institution just down the road that was also run by the Anglican Church of Melanesia. These RTCs are targeted at giving older youth practical business and job skills in basic construction, small shop management, local agriculture, and other such fields where a full education is not required.

The makeup and demographics of students at these schools is as equally complex as the different types of schools. Primary schools are always commuter-only for students. Extension schools (primary + year 7 and/or year 8) rarely offer boarding. Full junior secondary schools are usually commuter majority but also offer boarding for students. Senior secondary schools always offer boarding, which is utilized by a majority of students. All boarding schools do offer commuter options, however the population of secondary (and particularly senior secondary) schools is so large that only the cities (Port Vila and Luganville) have the transportation infrastructure necessary to support a majority commuter student population. The various types and compositions of schools is complex and pedantic but it is important to understand with regard to colleges, where students are usually sourced from a very wide geographic area and many different types 
of these schools. This information is available in the table below, for ease of understanding.

\begin{tabular}{|l|l|l|l|}
\hline School Type & Years Offered & Boarding? & $\begin{array}{l}\text { Majority } \\
\text { Boarding? }\end{array}$ \\
\hline Kindy & K & No & N/A \\
\hline Primary & $1-6$ & No & N/A \\
\hline $\begin{array}{l}\text { Extension } \\
\text { Primary }\end{array}$ & $\begin{array}{l}1-6,1-7, \text { or 1-8 (One } \\
\text { school) }\end{array}$ & $\begin{array}{l}\text { Rarely and only for } \\
\text { years 7 and } 8\end{array}$ & No \\
\hline $\begin{array}{l}\text { Junior } \\
\text { Secondary }\end{array}$ & $\begin{array}{l}1-6 \text { and } 7-10 \text { (Two } \\
\text { distinct schools) }\end{array}$ & Yes & No \\
\hline $\begin{array}{l}\text { Senior } \\
\text { Secondary }\end{array}$ & $7-12$ & Yes & Yes \\
\hline $\begin{array}{l}\text { College } \\
\text { 7-13 }\end{array}$ & Yes & $\begin{array}{l}\text { Yes, except in } \\
\text { Vila/Luganville }\end{array}$ \\
\hline $\begin{array}{l}\text { Rural Training } \\
\text { Center }\end{array}$ & $\begin{array}{l}\text { N/A - Trades, crafts, } \\
\text { business, etc }\end{array}$ & Yes & Yes \\
\hline
\end{tabular}

Primary schools usually have about one teacher per grade. Sometimes less, rarely more. These teachers can be all government paid or all locally paid or a mixture of the two. Government paid means that they are paid by the Teaching Service directly via direct deposit. No schools (primary, secondary, or college) are trusted to pay teachers directly, as this significantly reduces the money the government must give each school. In turn, this reduces the amount of money that can be mishandled. One trend to note is that teachers usually try to get teaching positions at schools in the area that they grew up in so that they can be closer to their families. A second trend to note is that because very few students in the most remote communities have the opportunity to receive the education to become a teacher, these communities rarely produce teachers. As a result of combining these two trends, the most remote communities naturally have difficulty filling open teaching positions, and as such they often go unfilled entirely and teachers are forced to teach multiple grades in the same classroom. Teachers cannot simply be 
assigned to these schools because the system by which teachers are appointed is corrupt and can be influenced very easily and there are not enough teachers to begin with. Junior secondary schools usually follow a very similar pattern as primary schools regarding teacher composition, up to and including all years of junior secondary.

Senior secondary schools or colleges, however, follow a slightly different pattern. This is because of the significantly higher number of staff required for the operation of a senior secondary school. Whereas primary schools theoretically have one teacher per grade, junior secondary schools theoretically have one teacher for each of 6-8 subjects and the same is true in senior secondary grades for each of 13 subjects. For instance, St. Patrick's College (SPC) had approximately 50 staff in 2015, composed of approximately 15 ancillary support staff, approximately 20 senior secondary level teachers (who sometimes also taught junior secondary grades/subjects), and approximately 15 junior secondary teachers. There is usually no funding for support staff at a school, which is why the SPC ancillary staff were paid directly by the Anglican Church of Melanesia. Because senior secondary schools are boarding and much more rare that primary/secondary schools, they are usually not tied to communities and the teachers are from all over the islands, including the occasional few that are foreign nationals, like myself.

In comparison to US high schools, there is very little similarity. The schools are most similar (yet still drastically different) to those of Britain, which follows naturally from Vanuatu's history with colonialism and the British empire. The similarities include heavily exam weighted grades, 13 years of instruction, and a relatively high number of 
boarding schools. In comparison to both British and US high schools, there is little to no similarity in education quality, subjects taught, or teaching methods.

\section{Fees, Logistics, and Availability}

The majority of people in Vanuatu do have relatively feasible access to primary education but significantly fewer have feasible access to junior secondary education. A very small percentage of youth have feasible access to senior secondary education. This is due to many things, like travel time/ability, value placed on education, and ability for students to succeed in schools. However the largest reason why students do not have access to education beyond primary years is simply money.

School fees are often a burden in primary school but are not so much that families (communities really) cannot afford them. Many primary schools operate without fees at

all, and technically primary education in Vanuatu must be free, by law. In reality this is simply not true. School fees in junior secondary schools range wildly, and are usually an even higher burden for families and communities. School fees in senior secondary are consistently very high. This is due to the heightened amount of infrastructure and staff required to operate these schools, the exam fees (to international education bodies and/or the Vanuatu Ministry of Education) that the school must pay, and the boarding of students for the entire school year.

Vanuatu has no taxes of any kind beyond a simple Value Added Tax of $20 \%$ to sold products, and thus the majority of its schools are grossly underfunded. Those schools that do enjoy a modicum of success are usually funded by external agencies or religious organizations. This funding, of course, comes with strings attached. For 
instance, at St. Patrick's College, students are required to attend 13 hours of church per week.

\section{Post Secondary Opportunities}

As one might imagine, opportunities for post-secondary education in Vanuatu are extremely limited. However there are three primary types: the teachers college, postsecondary trade schools, and university.

The teachers college is a post-secondary trade school where primary and junior secondary school teachers become certified to teach. It is located and attached to the most successful and prominent senior secondary school in the capital city and requires a year 12 leaving certificate ${ }^{5}$ from any senior secondary school for acceptance. The other postsecondary trade school is the Vanuatu Institute of Technology, which is also located in the capital city and also requires a year 12 leaving certificate from a senior secondary school for acceptance.

However, the main opportunity for a post-secondary education for most of the very few students that make it to senior secondary education, was and is the university. Specifically the University of the South Pacific (USP), which has campuses in many south pacific nations. The two attended most by Vanuatu's students are in the capital Port Vila, where a limited number of subjects are offered and in Fiji, where a far greater number of subjects are offered. Acceptance to USP requires a year 13 leaving certificate. Practically speaking for many students, it also requires passing at least 4 subjects' final exams with a 'Merit' mark (the highest) in order to qualify for a full scholarship.

\footnotetext{
${ }^{5}$ A leaving certificate is equivalent to a high school diploma except that it is leveled and therefore offered at all examinable years: $8,10,12$, and 13
} 
Without this scholarship there are still significant career opportunities for students that have IT and/or CS skills. These can include working for companies in Port Vila as a secretary or receptionist, at a bank as a teller, or doing non-computer based work for any of dozens of NGOs that require at least some computer skills occasionally. This, above all else, is the primary motivation for Vanuatu's education professionals and institutions driving IT/CS education at secondary schools even where the capacity for supporting such programs is limited, such as rural schools like St. Patrick's College. Following this aspiration is why Peace Corps is involved in these projects.

\section{History of St. Patrick's College ${ }^{6}$}

St. Patrick's College (SPC) is an Anglican Church institution and is one of the oldest schools in Vanuatu. In the early missionary days of the mid-1800s the Church of England took young men from Melanesia to New Zealand to train them as missionaries. After 30 years the school in New Zealand was relocated to Norfolk Island in Australia due to the inhospitably cold climate for Melanesians. Norfolk Island is geographically equidistant between Melbourne Australia, Auckland New Zealand, and Port Vila Vanuatu. In the early 1900s the Anglican Church of New Zealand decided to move and re-establish the school in Melanesia itself.

In 1903 a primary school was built at Vureas Bay on Vanua Lava island in the Banks island group of Torba Province in Vanuatu. Built by an Irish clergyman, Father McOferral named it St. Patrick's School, after Ireland's Patron Saint. The school remained at Vureas Bay from 1903 to 1935 before being moved to Nawalu Valley on

\footnotetext{
${ }^{6}$ The history of SPC is taken directly from a primary source, the Academic Principal during the time that I served there, Kathy Tahi. This source is located in the Appendix.
} 
Ambae Island, where it remains today. Among the many reasons for this move were problems with malaria-related death of staff/students, lack of fertile farmland to feed the school's occupants, and lowering the cost of the school's operation by moving it closer to the center of the country.

When the school added junior secondary years following a major renovation in the 1970s, it became known as both St. Patrick's School and Vureas High School. To this day, despite having no meaning on Ambae, "Vureas" is the school's most commonly used name. As the school slowly phased out the primary program, it began to develop the college years and eventually in 1996, it offered year 11 and 12 for the first time and became known as both St. Patrick's College and Vureas. Finally in 2001, year 13 was added which completed the school as the fully qualified St. Patrick's College that it is today.

\section{St. Patrick's College: A School of Two Thoughts}

SPC works like most schools in Vanuatu today. As a boarding school, the students have work, study, social, and personal responsibilities. The staff also live on campus and are composed mostly of Ni-Vanuatu teachers. There are approximately 400 students, 35 teachers, 15 ancillary staff, and 5 administrators who also teach. The Pacific Senior Secondary Certificate for years 7-12 (PSSC) and South Pacific Form Seven Certificate for year 13 (SPFSC) prescription ${ }^{7}$ systems are taught. This, however is where the basic similarities to other schools in Vanuatu ends. This is primarily because of the influence, history, and money of the Anglican Church.

\footnotetext{
${ }^{7}$ A prescription is simply the outline of a full curriculum. PSSC (year 12) and SPFSC (year 13) are prescriptions, which only cover the topics required for the final examination.
} 
The Anglican Church is the primary controller of the school. This is arranged through a Memorandum of Understanding with the Ministry of Education that gives an overall power structure split of approximately $60 / 40$ in favor of the church. This follows naturally from money. The Anglican Church provides for the entire operational cost of the school, including the cost of all ancillary staff, community teaching staff, parts/supplies, repairs, generator fuel, the school's truck, and additional special budgetary items like the cost of attending and participating in the annual provincial sports tournament. The government only provides for the salaries of the fully qualified government teachers (about 25-30) via direct deposit.

This system, while providing SPC with a higher level of funding and infrastructure than almost any school in Vanuatu enjoys, also leaves a constant power struggle at play. It is very common for the goals of the Anglican Church and goals of the Ministry of Education to come in to conflict. Inevitably, money wins and the Church makes the final call. These political dynamics are important to understand when assessing the viability of project stakeholders to work together, as demonstrated in chapters 4 and 6. They are also important to understand in the context of the sustainability/success of the school over time.

\section{History of the IT Program at St. Patrick's College}

The IT program at St. Patrick's College grew over a 4 year period beginning in 2007 from just a few computers, to some informal classes, to formalized instruction. By 2010, a Peace Corps volunteer was requested to teach year 12 computer studies classes according to the Pacific Senior Secondary Certificate prescription. That Peace Corps 
Volunteer (Melissa Burns) arrived in late 2010 and began teaching/training in February of 2011. Her work over the course of her standard Peace Corps contract (27 months) included the initial setup of curriculum resources, setup of a teaching-oriented computer lab, and finding a counterpart to train among many other things. By the end of that contract, she had expanded the year 12 prescription down into year 11 . This was primarily because the year 12 prescription covered far too many topics, skipped over necessary information, and assumed a greater exposure to computers than could be rightfully assumed for Ni-Vanuatu students. For instance, year 12 instruction begins with typing. This seems reasonable for brand new students, but not so much when one considers that typical Ni-Vanuatu students have never seen (and are very afraid of) computers. Thus a much slower introduction is necessary.

During her second year of service, Newman Tahi, the husband of the Academic Principal, became interested in Computer Studies at the school. Having a hardware certificate from the Vanuatu Institute of Technology, he was more than qualified to at least play a role in (even if not to teach) full classes. Over time Melissa began to work with Newman and teach him about the topics involved. She taught him the rudimentary aspects of teaching mechanics like lesson planning and course structuring. However, the majority of Newman's role during this time was learning for himself. It should also be noted, that as Newman was male and Melissa was female, such barriers often create a difficult work environment for Ni-Vanuatu people. This is particularly true for a group of just two people where one is female and the other is male. The gender roles, expectations, and cultural norms simply do not promote success in such an environment in Vanuatu. 
At the end of Melissa's standard 27 months of service, the South Pacific Board for Educational Assessment $\left(\mathrm{SPBEA}^{8}\right)$ released the first ever year 13 prescription. This prescription was and is extremely advanced, covering things ranging from website development and computer ethics up to and through Python programming. Knowing that no teacher in Vanuatu could hope to teach these subjects and that only two schools in the country offered the Computer Studies course at year 12 level put her in a very difficult position. She could extend her contract or leave the year 12 students that she had nurtured from year 11 upwards without a teacher for year 13. For these students this was the opportunity of a lifetime because IT/CS skills are in such short supply in Vanuatu. Even without a post-secondary education, there are lucrative IT/CS related careers and job opportunities available in the capital city for those students that want them. As a result, she chose to extend her contract for one more year. This also gave the Peace Corps time to find a replacement volunteer qualified to teach such advanced topics as those included in this brand new prescription.

During her third year of service, Melissa worked to understand concepts that she originally had little to no understanding of herself. Her background was in Journalism and Audio/Video Production. She created curriculum materials for teaching and all of her year 13 students passed their examinations. In fact, 9 students earned scholarships by

\footnotetext{
${ }^{8}$ SPBEA is a group in Fiji that creates the South Pacific Form Seven Certificate (SPFSC) prescription for all subjects and years. The Pacific Senior Secondary Certificate (PSSC) is simply the name given to the exact same prescription when it is adopted and administered by the Ministry or Department of Education in one of the nations that participates in the SPBEA. For Vanuatu, the year 12 SPFSC and PSSC prescriptions are identical and all schools are required to use the PSSC prescriptions for all subjects and years for which it is available, as administered by the Vanuatu Ministry of Education. However, since the year 13 SPFSC prescription has yet to be adopted for administration by the Vanuatu Ministry of Education, and thus there is no year 13 PSSC prescription yet.
} 
passing not only her subject but at least 3 others and went on to attend the University of the South Pacific.

At the conclusion of her service, a very large set of curriculum materials existed covering various topics to various degrees. Little digital organization existed for these items as many of them were on paper due to a lack of power (caused by a broken generator) for 6 months during her third year of service. In addition, Newman Tahi was more comfortable than ever in the classroom and what was once a disorganized mess of a few computers was then an organized lab of 15 workstations. These workstations had been pieced together from many broken and half-functioning computers, but they served their purpose well enough for the PSSC and SPFSC prescriptions to be taught successfully at that time.

As Melissa left it, the IT/CS education program at SPC consisted of 3 years of instruction. During year 11 students are required to take all 13 senior secondary subjects for 2 hours per week. This proceeds for 6 weeks, at the end of which students are required to choose 1 of 4 tracks to follow. The four tracks are: Life Sciences, Earth Sciences, Commerce, and History. At this point, students are also required to choose their one elective, of which there are 3: Agriculture, French, and Computer Studies. Due to the overwhelming demand to take Computer Studies, but the limited capacity for students, they are required to test in to the course based on two factors weighted equally: what they learned in those 6 weeks and why they want to take the course. The top 11 female and top 11 male students for a total of 22 students $^{9}$ were accepted. There are approximately 50 to

\footnotetext{
${ }^{9} 22$ was the capacity for the lab in 2014 and 2015, however with the new computer lab (as discussed in chapter 6) that number has most likely increased dramatically to 35 or more.
} 
60 students in each year 11 entering class, allowing for approximately $1 / 3^{\text {rd }}$ of students to participate in the IT/CS education program at SPC. Overall, the classes the students took at the senior secondary level looked like the table below. It is important to note that, while all students start with 6 subjects, failing the end of year exam for a subject prevents that student from continuing with the subject the following year. If a student drops below 4 subjects after year 11 or year 12 examinations, they are no longer permitted at the school for the following year. This is because 4 is the minimum number of subjects examined at the 'achieved' level required for entrance into the University of the South Pacific (USP). Also recall that 4 subjects at the 'merit' level (highest possible mark) are required for a scholarship to USP.

\begin{tabular}{|c|c|c|c|}
\hline Life Sciences & Earth Sciences & Commerce & History \\
\hline Mathematics & Mathematics & Mathematics & Mathematics \\
\hline English & English & English & English \\
\hline Chemistry & Chemistry & Development Studies & Development Studies \\
\hline Physics & Physics & Accounting & History \\
\hline Biology & Geography & Economics & Geography \\
\hline \multicolumn{4}{|c}{ Agriculture, French, or Computer Studies } \\
\hline
\end{tabular}

\section{Student Socio-Economic Backgrounds and Demographics}

When students first arrive at St. Patrick's College it is usually in year 7, however sometimes students don't come to SPC until later years to avoid the high fees of a college. SPC is a bit of a rarity in that it is both a rural and an advanced school. This makes it very attractive to students from all over the islands ${ }^{10}$ because it is not expensive (either in fees or in the cost of living) but yet it is ranked academically as the second best school in the nation. I would estimate that approximately $50 \%$ of SPC students are from Ambae island, another $20 \%$ are from the remainder of Penama province, another $20 \%$

\footnotetext{
${ }^{10}$ It is also attractive to parents in Port Vila SPC (being on the island, not in town) allows their child to retain a bit more culture and Kastom than strictly growing up in the city.
} 
come from Luganville/Santo and Port Vila/Efate, with the last 10\% coming from various places throughout the islands. Very few schools in Vanuatu pull students from more than a few islands, rather less most of them. This presents serious challenges for SPC to overcome.

These challenges usually take the form of students with drastically different backgrounds but also include logistical student travel difficulties. Very few students (like those from Port Vila) have had internet in their lives. Others (like those from the Banks) are at SPC on New Zealand based scholarship money, have never seen a computer, and their families have no money to speak of. SPC gets these extremes and everything in between. With this variation in home-location, comes a variation in many other factors:

- Internet Exposure

- Computer Exposure

- Exposure to and Knowledge of Jobs in IT/CS

- Family/Community Money

- Mobile Phone Ownership, Usage, and/or Exposure

- Primary Schooling Quality

- English Proficiency Level

Internet exposure is a newer socio-economic divider in Vanuatu, which makes sense since the internet itself is new there. Students from the city might have some limited (usually mobile phone) exposure to it. The same is true for computer exposure, however, very few students (even those from the capital) have had any real/meaningful exposure to IT/CS jobs or career role models of any kind. This means, when they get to SPC, they have to choose whether or not to take Computer Studies without having any idea what computers are actually good for. (This challenge was mitigated with good curriculum choices.) 
Primary schooling education quality levels vary drastically throughout the country and means that the students arriving at SPC in years 7-11 looking to join the CS/IT program are racing from very different starting lines. Nowhere is this more evident in the CS/IT program than in student's various English proficiency levels. Some students have excellent skills, like the children of SPC staff and others have very poor skills like the students from extremely remote villages. In many of these villages, it can be so remote that the adults do not even speak Bislama, only local languages. There is little to no standard as to how long students have been speaking English, ranging from 3 to 10 years on arrival in year $11 \mathrm{CS} / \mathrm{IT}$.

There is, however, one common thread among all Ni-Vanuatu students. This thread is with regard to the money they eventually make in any job they might end up getting. As with all things in Vanuatu, that money is collectively owned, meaning that large portions of it are sent back to family and communities. It is not uncommon for a Port Vila based worker to be supporting more than 10 individuals in their extended family. This is both culturally expected and in many cases a direct exchange/payback for a community/family pooling resources to send students for schooling. This is also a powerful driver to succeed at school for some students. It can often be the case that a village could only afford to send a single student to school through year 13, placing an enormous amount of pressure on that student to become the sole source of monetary income for a community. 


\section{Arrival of a New Peace Corps Volunteer at SPC}

Upon arriving in country in January 2014, I was immediately placed in a training village for 10 weeks so that I might learn the local language, customs, and various other things necessary to be a successful Peace Corps volunteer. I completed training in late March and arrived to St. Patrick's College (SPC) on April 1 ${ }^{\text {st }}, 2014$. As recommended over and over again during Peace Corps training, I spent the first 2-3 months actively attempting to learn about my site, its people, its history, and how things operate. This was invaluable beyond what words can express and (in retrospect) I did a very poor job of it. This was primarily a function of my arrival date to the school, which was two months into the school year and only 3 weeks from the end of the first term. I also discovered that my counterpart was many weeks behind schedule when I arrived. Thus, the immediate thrust in to work allowed me no time to just learn about my site and integrate. I did eventually accomplish this integration over time, but had I not done so my service, projects, and experience overall would have been significantly less successful/enjoyable.

This is an important point to note about the differences between volunteer organizations that work on the grass roots level, those that don't, and top-down development organizations. For example, missionary work in Vanuatu had very significant impacts on the country and this was a form of top-down development. The purpose was to change Vanuatu and its people to be the missionaries' perspective of better. Whether that meant changing religions, subjugating women, enforcing strict gender roles, or adapting the social norms for things like 'appropriate' clothing. All of these things happened in Vanuatu. In addition, many other organizations (even those that do serve on the grass roots level) have little to no cultural or development training. This 
leads to situations in which volunteers, organizations, and money all promote approaches (intentionally and otherwise) that are not only unsustainable in Vanuatu, but also destructive of Vanuatu's culture, history, and traditions. The challenges that I examine in this report with regard to the projects that I worked on during my Peace Corps service are not problems. They are challenges. This distinction is the difference between using a different approach versus attempting to find a solution. Very rarely during my experience was a challenge easily solved with a solution. Rather, a culturally sensitive approach to accomplishing their goals was the only methodology that worked and it is the only methodology that Peace Corps supports. To as great an extent as I could manage, I followed this methodology. However, there were occasions in which Vanuatu's culture and the nature of IT/CS education were at odds. At these times, a compromise that prioritized their goals, even if it required a departure from their cultural norms, was the most effective approach. As such, it must be noted that some of these approaches might have unintended change-their-culture type consequences.

\section{Throughout Service: Internet, Electricity, and the Computer Lab}

The computer lab facilities, internet, and electricity varied somewhat throughout my service. When I first arrived at SPC in April of 2014, there was a satellite linkup to an Australian company. It provided $56 \mathrm{kpps}$ at a cost of $\$ 319$ per month. This system went down by November because the principal decided not to pay the bill. Following this, internet was not restored at the school until after my service completed in September 2015. Digicel (a local phone provider) did complete installation of expensive and poor quality but relatively fast $3 \mathrm{G}$ internet in May of 2015. Due to the cost and poor signal (from within a giant, volcanically formed valley) this solution was rarely used for ICT at 
the school, however, it was supposed to be the solution included with the grant program for a new computer lab as discussed in chapter 6 . To date, I am unaware of its successful implementation with the rest of the computer lab.

The computer lab itself functioned surprisingly well during my time at SPC. I started with 8 separate desktop computer stations and 14 n-computing ${ }^{11}$ stations. By the end of my service, only 12 n-computing stations remained. Electricity on the other hand, was not reliable at all. The primary $(57 \mathrm{KW})$ generator for the school campus went down many times despite being brand new when I arrived. This was due to excessively poor maintenance. During my second year of service, following the March 2015 Category 5 Cyclone Pam, the generator was damaged and soon became non-repairable. This remained the case through the end of my service in September 2015. Backup generators were brought in, but they provided insufficient power to run the lab. Thus, class was cancelled and occasionally run with iPads donated from the Anglican Church.

\footnotetext{
${ }^{11} \mathrm{~N}$-Computing is a virtualization software that creates multiple Windows instances on multiple monitors/keyboards/mice using the Local Area Network.
} 


\section{Chapter III: Curriculum Development and Improvement}

There were primarily 5 projects that I worked on during my Peace Corps service, as listed below. The order that they are discussed in is not proportional to the amount of effort given. Instead they are ordered for the mechanics of the reader's understanding. There is prerequisite knowledge about each project that is helpful to learn from the projects discussed before it. In the Peace Corps model, teacher training and capacity building (from the perspective of sustainability, institutional memory, teaching quality, etc) are the top priorities. Secondary priorities were providing direct aid and resources like computer labs as well as Gender and Development (GAD) work.

These projects were:

1. Curriculum Development and Improvement

2. Teaching Classes

3. SPC Staff Capacity Building

4. Computer Lab Project

5. Gender and Development Project

\section{Project Overview}

During my service, one of the major projects that I worked on was creating year 11/12 Computer Studies ${ }^{12}$ and year 13 Information and Communication Technology (ICT) curriculum materials for use in three ways: teaching classes myself where necessary, training my counterpart, and providing for the sustainability of the SPC IT program. These curricula were created based on the requirements laid out in the prescriptions, which define what the students are examined on in years 12 and 13. Each prescription has both Internal Assessments (IAs) and an External Assessment, which is

\footnotetext{
${ }^{12}$ Note that CS does not refer to the name of the year 11 and 12 class Computer Studies. Throughout this report, CS always refers to Computer Science.
} 
just the final exam. The internal assessments are a required series of pre-approved projects of the teacher's design or provided assessment forms to be filled out by the teacher for each student. There is one IA for each of the chosen topics, depending on the prescription's subject and year. Samples, copies of, or just the teacher's grades are required to be sent to the prescription authority by various deadlines throughout the year. For year 12 Computer Studies there are 3 project-based IAs and for year 13 ICT there are 5 IAs, composed of 3 project-based ones and 2 teacher completed assessment forms. The basic structure of the year 12 PSSC prescription is below. Note that topics 1 and 2 are compulsory and form $70 \%$ of the required teaching. From the remaining optional topics, $30 \%$ must be chosen and taught at the instructor's discretion. Because the optional topics and the approved IA projects are required to be changed by each teacher every 2-3 years, I worked to develop and improve curriculum materials for all of them.

\section{Year 12: Computer Studies Prescription (2007)}

\section{Topic 1. Compulsory - Desktop Computer Systems and Management (35\%)}

Objective 1.1 Describe, operate, and maintain the hardware components of a desktop computer

Objective 1.2 Describe the purpose and functions of the operating system

Objective 1.3 Describe the purpose of the keyboard

Objective 1.4 Operate a desktop computer printer

Objective 1.5 Describe how computer data is stored

Objective 1.6 Describe desktop computer software

Objective 1.7 Describe the graphical user interface (GUI)

Objective 1.8 Locate, manage, and protect computer files using file management procedures

Objective 1.9 Explore legal, ethical, and social issues related to accessing data held in computer systems

Objective 1.10 Know the key roles and responsibilities of people in the field of information and software technology

Topic 2. Compulsory - Using Personal Computer Applications (35\%)

Objective 2.1 Demonstrate an understanding of word-processing principles and terminology

Objective 2.2 Load and exit a word-processing application and use the menu and toolbar 
Objective 2.3 Use word-processing principles and functions to enter, edit, and format a document

Objective 2.4 Demonstrate knowledge and uses of spreadsheets

Objective 2.5 Produce a simple spreadsheet file containing labels, values, and mathematical formulae

Objective 2.6 Manage spreadsheet files

Objective 2.7 Manipulate the data in the spreadsheet

Objective 2.8 Demonstrate knowledge of the uses and features of databases

Objective 2.9 Manipulate data in a database

Objective 2.10 Manage database files

Objective 2.11 Exporting/importing data between computer applications

Topic 3. Optional - Programming Computers (20\%)

Topic 4. Optional - Desk Top Publishing (10\%)

Topic 5. Optional - Data Integration Between Computer Applications (10\%)

Topic 6. Optional - Computer for Leisure and Learning (10\%)

Topic 7. Optional-Computer Presentations (10\%)

Topic 8. Optional - Computer Graphics (10\%)

Topic 9. Optional - Using E-Mail (10\%)

Topic 10. Optional - Keyboarding Skills (10\%)

Topic 11. Optional - History of Computing (10\%)

Topic 12. Optional - Internet Research (10\%)

This prescription is now split into two years as the two full curricula shown

below. Each unit has a full catalog of resources including PowerPoint presentations,

homework assignments, project rubrics, handouts for further learning, videos where

appropriate, in class assignments, notes, readings, and many other materials.

\section{Year 11: Introduction to Computer Studies Curriculum}

\begin{tabular}{|c|c|}
\hline Unit 0 Do You Want To Take Computer Studies ${ }^{13}$ & 6 Weeks \\
\hline Unit 1 Welcome to the Lab & 1 Week \\
\hline Unit 2 The Mouse & 1 Week \\
\hline Unit 3 The Keyboard and Typing & 2 Weeks \\
\hline Unit 4 Basic Hardware & 2 Weeks \\
\hline Unit 5 The Windows User Interface & 2 Weeks \\
\hline Unit 6 The Culture Map (Mastering the Mouse) & 2 Weeks \\
\hline Unit 7 Software Types & 1 Week \\
\hline Unit 8 The Operating System & 2 Weeks \\
\hline Unit 9 Memory, Storage, and Data Processing & 2 Weeks \\
\hline
\end{tabular}

\footnotetext{
${ }^{13}$ Note that Unit 0 in year 11 refers to 6 weeks of instruction over all senior secondary subjects before students have chosen which track of subjects they would like to proceed with.
} 


\begin{tabular}{|l|l|}
\hline Unit 10 The Filesystem and Best Practices & 2 Weeks \\
\hline Unit 11 Networks and the Internet & 1 Week \\
\hline Unit 12 Microsoft Word & 4 Weeks \\
\hline Unit 13 Microsoft Excel & 7 Weeks \\
\hline
\end{tabular}

\section{Year 12: Computer Studies Curriculum}

\begin{tabular}{|l|l|}
\hline Unit 1 Welcome to the Lab & 1 Week \\
\hline Unit 2 Review Mouse, Keyboard, and Typing & 2 Weeks \\
\hline Unit 3 Review Basic Hardware & 2 Weeks \\
\hline Unit 4 Review Software, Memory, Storage, and Data & 2 Weeks \\
\hline Unit 5 Review Operating Systems and Windows UI & 2 Weeks \\
\hline Unit 6 Graphical User Interfaces & 2 Weeks \\
\hline Unit 7 Printers & 2 Weeks \\
\hline Unit 8 Filesystem, Security, and Backup & 3 Weeks \\
\hline Unit 9 Social, Ethical, and Legal Issues in ICT & 2 Weeks \\
\hline Unit 10 Careers in ICT & 1 Week \\
\hline Unit 11 Microsoft Word Newsletter Project & 3 Weeks \\
\hline Unit 12 Review Microsoft Excel & 2 Weeks \\
\hline Unit 13 Databases and Microsoft Access & 5 Weeks \\
\hline Unit 14 CAT Practice and Exam & 2 Weeks \\
\hline Unit 15 Email & 2 Week \\
\hline
\end{tabular}

The basic structure of the SPFSC prescription is much higher level and simpler, primarily because it is 7 years newer than the PSSC prescription:

\section{Year 13: Information and Communication Technology Prescription (2013)}

Unit 1. Open Source and Proprietary Software

Topic 1.1 Software Sources

Topic 1.2 Installation

Topic 1.3 Recommendation

Unit 2. Media

Topic 2.1 Graphics

Topic 2.2 Video

Topic 2.3 Audio

Topic 2.4 Integrated Media Output Project

Unit 3. Ethics of ICT

Topic $3.1 \quad$ Piracy

Topic 3.2 Security

Topic 3.3 Privacy

Topic 3.4 Copyright

Topic 3.5 Longevity of Information Held in Electronic Form

Topic 3.6 Intellectual Property 
Unit 4. Programming

Topic 4.1 Design

Topic 4.2 Coding

Topic 4.3 Testing

Topic 4.4 Project: Create your own code

Unit 5. Environmental Issues and Climate Change

Topic 5.1 E-Waste

Topic 5.2 Power

Topic 5.3 Raw Materials

Topic 5.4 Sustainability

Topic 5.5 Climate Change

Unit 6. Website Design and Development

Topic 6.1 Design

Topic 6.2 Development

Topic 6.3 Testing

Topic 6.4 Project: Create your own website

Unit 7. Safe Practices

Topic 7.1 Health

Topic 7.2 Identity Theft

Topic 7.3 Physical Security

Unit 8. Microprocessor Control

Topic 8.1 Microprocessor Hardware

Topic 8.2 Software Creation

Topic 8.3 Embedded Microprocessors

The year 13 ICT prescription did not require anywhere near as much

reorganization work, but it did require a much larger amount of resource development and teacher training. This was primarily because the topics are so much more difficult. The final curriculum for year 13 is shown below. This curriculum also contains a full catalog of various resources for each unit.

\section{Year 13: Information and Communication Technology}

\begin{tabular}{|l|l|}
\hline Unit 1 Open Source and Proprietary Software & 2 Weeks \\
\hline Unit 2 Media & 4 Weeks \\
\hline Project: Instruction or Music Videos & 3 Weeks \\
\hline Unit 3 Ethics of ICT & 2 Weeks \\
\hline Unit 4 Programming & 5 Weeks \\
\hline Project: Hangman or Tic-Tac-Toe & 5 Weeks \\
\hline Unit 5 Environmental Issues and Climate Change E-Waste & 2 Weeks \\
\hline Unit 6 Website Design and Development & 4 Weeks \\
\hline
\end{tabular}




\begin{tabular}{|l|l|}
\hline Project: Personal Website & 3 Weeks \\
\hline Unit 7 Safe Practice & 2 Weeks \\
\hline Unit 8 Microprocessor Control & 5 Weeks \\
\hline
\end{tabular}

The largest value that I was able to provide in developing curriculum for SPC's IT/CS education program was from my background and prior knowledge in IT/CS. This allowed me to spot problems with the prescriptions and attempt to fix them in the curriculum as best I could. It also allowed me to make better decisions than someone experienced only with general education with regard to IT/CS because I had a greater core knowledge and deeper understanding of the concepts. As Einstein put it: "You do not really understand something unless you can explain it to your grandmother" [11]. This benefit manifests in many, but most notably 5 ways:

First is that a core understanding on its own allows for better explanations. For instance: I was able to explain why the ordering of words in a Python statement mattered. This required an understanding of how compilers worked as well as an excellent level of comfort with terms like identifier, variable name, type, etc. A teacher who had learned these things on their own would have resorted to simply specifying how the syntax works instead of why it works that way in the curriculum materials and resources. I made this exact change to some slides that I had inherited from the previous volunteer.

Second is being aware of vital prior knowledge and/or experience that is missing, both in the students and the prescription. For instance: There is nothing in the prescription about how to use the Windows operating system's User Interface (UI), like the start menu, taskbar, system tray, desktop, and Windows Explorer application. This is both essential prior knowledge as well as essential prior experience. Having a deeper 
background in IT/CS allowed me to be aware of the cases (like this) where knowledge is no substitute for experience and vice-versa. I thus created a curriculum module specifically for the Windows UI that required students to follow written instructions like:

"Open the start menu and hover over the All Programs menu until it opens, then open the Microsoft Office folder."

Understanding the written instructions helped them learn the knowledge (particularly vocabulary) and following them actually gave them the experience in knowing what the many Windows UI operations look and feel like so that they became more comfortable with them. A teacher with less background in IT/CS might not be aware that interpreting the GUI's subtle feedback like shadows and selections and highlights is a learned skill.

Third is knowing the order to organize topics within the curriculum. Similar to when topics might be missing from the prescription or student's knowledge altogether, teaching topics in the wrong order might exaggerate a prerequisite knowledge gap unnecessarily. For instance: The year 12 prescription requires a Common Assessment Task (CAT) that is essentially a one hour practical exam on Microsoft Word, Excel, and Access. Because of the timing with Vanuatu's holidays it is very easy for a teacher to make the mistake of trying to teach Access first or rush through Excel in order to get to Access. This is also because the exam is mostly on Access. However, the mistake here that a teacher not thoroughly grounded in CS/IT principles might make is that a relational database (at its most simplistic) is a bunch of related tables. Students have no hope of understanding them without understanding tables first. Thus, Excel must come first and be thoroughly covered before Access, which would then be a naturally easier unit. 
Fourth is a better understanding of knowing what to, what not to, and what level to...teach a concept in the first place. For instance in what to teach: Something that should definitely be taught to the year 12 students but was not in the prescription, nor was it prerequisite knowledge for a topic that was in the prescription, was how to set up a home router. This is an important skill because nearly all internet in Vanuatu is wireless. Understanding the router's function and being able to configure one or set it up are two very different things. A different teacher might assume that knowledge of a router and how it functions is enough to figure out how to set one up. This is a developed world ethnocentric assumption.

For instance in what not to teach: Floppy drives, serial/parallel ports, and PS/2 ports were not in the prescription but were in the curriculum that I inherited. While these are great and fun things to learn about and seem important to a less CS/IT experienced teacher, they are completely irrelevant in Vanuatu. The vast majority of computers in the country are laptops, and on the very rare occasion a student might need to learn such things they can learn it on their own. They are not hard concepts but creating entire curriculum materials for them is a waste of otherwise valuable class and curriculum creation/maintenance time.

For instance in what level to teach: The curriculum that I inherited taught both Object Oriented Design and its practice. This was a mistake. The course is not designed to go deep enough into programming to warrant anything more than a high-level basic understanding of the idea, if that! Attempting to have students figure out variables, types, simple data structures, syntax, and logical flow of instructions all for their first and only 
programming assignment is a huge cognitive load on its own. Having undergone a formal CS education, I knew better and immediately removed the object oriented implementation aspects. As another example of the level to teach concepts is the databases unit, where teaching about non-relational database technologies was useless and too much of a cognitive leap for students that struggle to understand relational ones in the first place. I again, immediately removed it, knowing that non-relational databases are not foundational.

Fifth and finally is knowing not just what to teach, when to teach it, or the level to teach it, but how to teach it. For instance: My counterpart really liked using PowerPoint presentations. These are sometimes a very effective tool, but he used them incorrectly by reading them aloud and putting far too much text on them. He also used them when they weren't the best tool for the job, as was the case in teaching how binary works. Instead, we created a teaching notes document and used the whiteboard ${ }^{14}$ instead so that we could have a flowing example with constantly changing bits. This example gets into my teaching and teacher training projects, which are discussed in chapters 4 and 5 . These projects naturally have a lot of crossover, but the point of this example is to demonstrate that PowerPoint is not always the best tool in curriculum development and it often takes a teacher with a deeper core understanding of the topics to see that before attempting to teach it.

\footnotetext{
${ }^{14}$ Note that the vast majority of Vanuatu classrooms do not have access to whiteboards or whiteboard markers. These markers had to be couriered from the capital whenever someone went. Most schools use chalkboards, which are of course terrible for computers due to the dust.
} 


\section{Challenges: Prescriptions}

The prescriptions themselves presented many problems that become immediately apparent in trying to develop effective and sustainable curricula. I suspect that this is the result of a lack of qualified professionals working on the curriculum at the SPBEA.

Above all are six issues:

First is the cognitive overload from requiring too many topics too fast, for example covering all of Microsoft Office (Word, PowerPoint, Excel, and Access) within only the year 12 prescription's $2^{\text {nd }}$ compulsory topic. This topic (if following the prescription) would then be taught in a single term of 13 weeks.

Second is poor teaching order of topics, like teaching Environmental Practices in between Python Programming and Web Development. Python Programming and Web Development are both programming units. To put a theory unit that requires a great deal of conceptualization (from the perspective of Ni-Vanuatu students) in the middle of two programming units, requires not one but two cognitive leaps when only one is necessary.

Third is incorrect priority levels for topics, as was the case in giving equal weight to the two compulsory topics in year 12 when they should probably be more like a 30/70 split. The first topic is about what computers are made of, how to use them, and the basics (hardware and software) of how they operate. The second is all of Microsoft Office. At this stage of their CS/IT education, Microsoft Office is far more important.

Fourth is the disconnect between the year 12 and 13 prescriptions. There is virtually no overlap, connection, or natural flow from one to the next. This is partially a product of being 7 years separated in release date. The year 12 prescription in a nutshell 
is about hardware, software, user skills, and Microsoft Office. Year 13 however is primarily a combination of Computer Science theory/ethics as well as programming.

Fifth is the lack of many important topics in the year 12 prescription that leave gaps of prerequisite knowledge for teaching other topics. For instance the year 12 prescription requires learning all about how computers operate and what their functions are but places very little emphasis on the prerequisite knowledge and skills of being able to actually use a computer first.

Finally was the outdated nature of the prescription and its topics, particularly for year 12 which was developed in 2005 and 2006 for release in 2007, meaning that (at the time) it was anywhere from 7 to 10 years out of date. Concepts like floppy drives and serial/PS2 ports were given full exam questions and yet ideas like the Blu-Ray and the Internet of Things did not exist.

\section{New Approaches: Prescriptions}

The cognitive overload challenge presented by the year 12 prescription was mostly mitigated by expanding the curriculum down into year 11. I expanded it further down into year 10 at the beginning of my service but this proved logistically impossible and did not end well. I had not done my research properly and in an attempt to please their new Peace Corps volunteer, SPC's staff accommodated my request without informing me that it required the year 10 students to give up 2 hours of another subject. The teachers collectively chose French as less important and it was downgraded to 2 hours per week. Upon finding out, I insisted that year 10 IT/CS should be discontinued

for the $3^{\text {rd }}$ term and onwards. I had failed to be aware of my privilege as a white 
American male, and the same request would never have been accommodated for any other teacher. For year 13, the cognitive overload challenge wasn't really solved and I can't imagine any successful solution without a new prescription. There is simply too much to cover in a single year, and as such I did not complete the teaching of the course during either year of my service. During both years, I did not even start teaching the final Microprocessor Control unit. Thankfully, the unit was usually not given much weight on the student's final exam for exactly this reason.

Improving the ordering and priority levels of teaching topics was a simple fix, but it requires a deeper core understanding of CS/IT principles than most teachers would have. I simply had to reorder the topics and spend more/less time on them. The one caveat is that in order to do this, Internal Assessment deadlines had to be ignored. This generally wasn't a problem since deadlines really don't matter in Vanuatu anyway.

I never found an approach to the challenging disconnect between year 12 and 13's prescriptions. I am not sure there is one because the prescriptions and their associated final exams were completely out of my control.

A new approach to help mitigate when students lacked important prerequisite knowledge and the outdated information in the year 12 prescription was partially implemented before my arrival. This was the expansion of the year 12 prescription in to both the year 11 and 12 curricula. However, both of these challenges require a core understanding of IT/CS issues. Thus I was able to make some changes to improve things further; most notably by adding an entire curriculum unit to year 11 focused on practicing Windows UI skills. 


\section{Challenges: Work Environment}

Working in the physical environment of SPC is not too great of a challenge, but to do so usually meant a significantly greater amount of effort with lesser results compared to a typical office or work environment in the developed world. This is due to constraints like electricity, air conditioning, excessive heat and humidity, and lack of quiet workspaces. In addition, the lack of internet also placed a cap on what gains could be made regardless of mine or my counterpart's persistence through this environment.

\section{New Approaches: Work Environment}

The approach that I found was best for getting through the difficult working environment was to accomplish a great deal of work in the capital city, where electricity, air conditioning, and internet were readily available. Whenever I was in the capital ${ }^{15}$ for any reason I always set aside some time to look up information, download videos, and make new resources for classes. The more comfortable environment (for me and the computer) as well as the access to internet, yielded much higher gains for similar effort levels. This allowed me and my counterpart to simply edit, adapt, and review these resources together on the island, meaning significantly less work in a non-hospitable environment. This approach however, had obvious negative implications for the sustainability of the curriculum development project as well as the teacher training project because it required flights to/from the capital, which is obviously not feasible or sustainable.

\footnotetext{
${ }^{15}$ Peace Corps Volunteers go to the location of the PC in-country HQ with varying degrees of frequency depending on medial, safety/security, training, and other needs. On average I would guess that I spent 6-8 weeks at site for every 1-2 weeks in the capital. Sometimes as long as 4 months at site and other times as little as 3 days.
} 


\section{Challenges: UAI, LTO, and MAS}

I am of the opinion that the Uncertainty Avoidance Index (UAI) for Fiji is not representative of Vanuatu, however it is 48 compared to the United States' value of 46 [5]. There is no data for either Vanuatu or Fiji's Long Term Orientation (LTO), however from my own personal experience I would think it is very low. This is probably biased. As shown in chapter 2, my own personal LTO is significantly greater than that of my own culture, 69 vs. 26 respectively [5]. The biggest way in which these two HCDs affect curriculum development at SPC can be summed up with a single four letter word: time. Anyone who has done work in the developing world can tell you that time is drastically different. It varies by region but in many places it is known as island time and it is a very important concept to understand. Time is so different that it was very difficult to truly adapt to and is even more difficult to explain to others. For example, the common American expression 'time is money' is literally laughable in Vanuatu. I tried explaining it to some ancillary staff at the school once and it was like trying to explain the concept of a desert to a fish. The idea that time could have value is as alien to them as extraterrestrial beings. The implications of this are enormous. Lesson plans cannot be planned according to time. Course calendars cannot be planned according to time. Exams cannot be planned according to time. Cancelled classes become very common, but with a low UAI, planning in the curriculum for cancelled classes is difficult and problematic. This is because uncertainty is very normal to people in Vanuatu, and writing up plan a, b, and c in a curriculum or lesson plan is very unnatural for them. The first question many would ask about this is: Well, then how did it all get done, rather less get done on time? The answer is simple. It didn't. As a culture with a low Masculinity (MAS), accomplishing things 
was not as important, particularly when said things required a high LTO, like planning an entire year long course or 3 year IT program. Logistically speaking, a low LTO also created a difficult situation in just scheduling the meetings to create curriculum with my counterpart. Because these meetings had to take place outside of normal teaching hours, social obligations almost always took priority. Low LTO and low UAI were of course factors of great significance for both the curriculum development and teacher training projects.

\section{New Approaches: UAI, LTO, and MAS}

When I first started teaching I made the mistake of giving my students assignments with hard deadlines. While this works for small assignments that are easily understood, it does not work (at all) for projects, large assignments, or things for which there might be any difficulty. In my experience, this was due to a cognitive disconnect between something being too difficult and simultaneously due regardless. In a culture with low UAI, low LTO, and low MAS like Vanuatu, if some project in the village is difficult or is undergoing some unforeseen setbacks, then it will get done when it gets done. Period. End of story. This was how students approached difficulty in curriculum topics... wait and it will resolve itself. In my experience, it was much better to design projects with many check-ins built in throughout it. For example, one Internal Assessment (IA) that I gave to my year 13 students during my first year was to make a YouTube instruction video for some common task like washing laundry or making Kava. ${ }^{16}$ I gave them one hard deadline and that was it. Needless to say that not a single

\footnotetext{
${ }^{16}$ Kava is the local drink of choice. While not alcoholic, it does have a unique high-like effect on the drinker.
} 
student turned it in less than a month late after endless nagging. During my second year, the same project was to create a music video (in boy-girl pairs) where the voice of the each student was swapped ${ }^{17}$ while the other appeared in the video. But by breaking the project down into multiple deadlines for easier portions, I was able to check in with them much easier. Not a single one of the internal deadlines was hit, but it allowed the final deadline to be missed by only 1 week as opposed to 2 months.

More generally speaking with regard to lesson planning... the degree of uncertainty that can be experienced at SPC is enormous. I found it was best to simply not plan lessons and teaching methods. This is obviously controversial, but I found it worked better. Reviewing the material to be covered during the upcoming unit is definitely needed, but planning for each lesson was a practical impossibility. I originally started with a beautiful lesson plan template that accounted for learning styles, timing, objectives, methods, homework, and all the other things we typically think about when lesson planning in America. I tried (and failed) over and over again to force my counterpart into creating and writing such lesson plans. I also tried to use those same lesson plans in class when we did manage to make them. That also failed... horrendously. I tried a simpler one, and a simpler one after that. Both of them failed too. It took me a very long time to figure out why they didn't work, and it happened very slowly. Finally, during my second year, it clicked.

Keeping it simple was, is, and will always be the key for sustainability and success of anything in the developing world. Once you think you have it simple enough,

\footnotetext{
${ }^{17}$ This also combats gender role stereotypes and expectations, as further discussed in chapter 7.
} 
make it simpler. To that end, my counterpart and I tried making course materials that inherently did not require a lesson plan and were based on subject instead of time. We tried this with the "Introduction to Software" unit in year 11 by creating one giant PowerPoint that covered the entire topic. Much like how a University course sometimes operates, we would just go through the topic until the end of class and continue with it in the next class. From a course and lesson planning perspective, it worked like a charm. There were however two major drawbacks. The first was that if a single teaching method failed (i.e. - power was lost during a unit that uses PowerPoint) then backup teaching methods for the same material were needed. Thus, for all topics, not only did many redundant resources need to be created, but also maintained over time for the curriculum to be truly successful and sustainable. The second major drawback to this approach was that, because time was not part of the lesson planning process at all, it was also not part of the course planning process at all. What could be accomplished with the course timeline was a general idea, based on milestones. In each curriculum, there are a certain number of weeks assigned to each unit. This is a loose guideline. It can be affected by holidays (of which Vanuatu has a never ending list) as well as unforeseen cancellations, delayed starts of term, early ends of term, last minute exams thrusted upon the teacher, environmental conditions, cyclones, malaria outbreaks, etc. With a milestone-based course guideline, one can simply pick a teaching method for the topic and go. Simple $=$ Successful.

In addition to these drawbacks (from my perspective) there were also some correlative drawbacks in the teaching project that will be discussed in chapter 4 . 


\section{Challenges: IDV}

Low IDV drastically affects the learning environment. Whether for good or for bad is a matter of opinion. During my first year teaching I would often get assignments handed in that were identical but had different names. I would ask if the two (or more) students had worked on it together and they would say no. Eventually my counterpart explained to me that asking if they worked on it together was asking if they had handed in one assignment with multiple names, not if they had done the assignment separately. This also explained the strange looks students would give me upon me simultaneously holding physically separate assignments and asking this question. The very concept of doing an assignment without help from fellow students was unheard of. This was both because help from teachers was not easily available in or out of class and because the thoughts of a group are a fundamental input to the process of how Ni-Vanuatu students form their opinions and answers. Generally speaking, preparing for an exam in the Vanuatu education system is a function of collectively memorizing the truth as opposed to individually understanding the principles and synthesizing the required result for each question. This became extremely problematic for their final exams, since they were not created by Ni-Vanuatu teachers and required much higher levels of critical thinking and concept synthesis than students were comfortable or practiced with.

It should also be noted that the CS/IT subject is very different than other subjects at SPC. Other subjects taught at the school are not rapidly/recently changing, they require little to no hands-on work, and they can be taught by Ni-Vanuatu teachers who know the subject. Other subjects are usually taught with low-IDV methods, like copying notes from the whiteboard or read the book and write a summary. These aren't really curriculum 
methods, just teaching styles, because they aren't complicated enough to warrant a lesson plan. In fact, teachers often use no real plan at all. I always wanted to use high-IDV teaching methods like interactive Excel spreadsheets, but the students expect low-IDV methods like copying notes. In addition, because IT/CS education materials that already exist are largely from the developed world, finding low-IDV curriculum materials (in English) is difficult as well.

One of the primary concerns with collectivist thinking in curriculum methods is the subjugation of the individual's opinion in favor of the group's consensus, as was the case when the curriculum utilized any form of group work. This was particularly true when a student's opinion was drastically outnumbered within a group, compounded by gender roles, or when Vanuatu's high Power Distance Index was at play. A specific curriculum example that illustrates all three of these compound-factors to low IDV was Jeopardy Review. Jeopardy Review (the game show as a PowerPoint) was a review method that my counterpart really liked because it was fun. However, by splitting up the class into separate small groups, this often resulted in a group with 3-4 male students and a single female student. A male student decides on the answer but the female student would be uncomfortable disagreeing with the rest of her group, especially the male spokesperson for her group. Lastly, speaking to me (the teacher) in class (as the spokesperson) would be difficult for any student, but especially female students. The three combine for a horrendously uncomfortable environment for students. 


\section{New Approaches: IDV}

The first thing that I must say about low Individuality (IDV) is that individual thinking (and particularly critical individual thinking) is a foundational element to a learning environment. My counterpart and I agreed on this and made a conscious decision to change their culture (in class anyway) in this regard. I should note that this is controversial and I am not qualified to make such decisions. Regardless, I was forced to make the decision, so I chose and I stand by my choice and its rationale.

The approach that I found worked best in the case of low IDV was to plan for students to have mostly low IDV homework and projects, but a high IDV classroom environment. My counterpart and I did this because we could more easily control a classroom environment. PowerPoint presentations are an effective compromise when you have electricity because they are participatory when used on individual screens and excellent for students who might have vision problems ${ }^{18}$, but still allow students to follow along at their own pace. This makes it a middling IDV method, which they are mostly comfortable with. Vanuatu has a very high concentration in K-13 learning on rote memorization as the primary and often only method. This allows them to utilize the process that they are most comfortable with without having to copy endless trivial information from a whiteboard.

We also created curriculum and lessons that utilized the computers extensively via participatory methods. The subject itself helped with this tremendously. We were able to plan for the students to use the computers individually without making them feel

\footnotetext{
${ }^{18}$ Note that glasses are rarely afforded in Vanuatu due to cost. They are also rarely worn or sought out in the first place due to shame within teenage peer pressure groups.
} 
uncomfortable as they would during high IDV work in most any other subject. This is because high IDV work in other subjects might require student presentations or class discussion, but in IT/CS it only required that they use the computers on their own. The computer itself was a bridge to a higher IDV and critical thinking skills without the shame that is such a prevalent teaching method in Vanuatu. Despite our best efforts, sometimes students were put into uncomfortably high IDV situations by the curriculum methods we employed. The best that we could do in such situations was to be very aware of them and attempt to mitigate them with positive reinforcement and eliminating shame. Where curriculum could not help in preventing the difficulties that result from a low IDV environment, new approaches presented in the teaching project nearly always could.

Outside of the classroom, homework assignments could either allow for group work (in which they are far more comfortable) or they had to be explicitly designed to prevent it. One such assignment that I used required the students to write their own names in ASCII while learning about how data is stored. Since the answers are different, students naturally worked alone. This works in that students work independently, but for larger projects the students would still work together and simply do more work for the students that were struggling. I usually did not try to combat this for larger projects because the students still had to learn, just from a peer, which is of course a very powerful method of learning if they are learning. For instance, there is an assignment in year 12 while learning about file types where students are asked to make an A3 size poster (hand drawn) about a chosen file extension. Since each submission will look drastically different in both creativity and content, help from other students can only be in the form of learning as opposed to copying and/or rote memorization. 
There were methods for promoting high IDV that I tried that failed, usually horribly. Among them are two in particular: competition grades and classroom role reversal.

Competition grading was built into the assignment itself, where helping other students would hurt your own grade. I tried this with my year 13 students during my second year of teaching on their Python programming assignment. The student whose Hangman program had the most words got the best grade. I received 6 assignments, each differently implemented but every single one with exactly 13 words built into the game. In such a low IDV culture, loyalty and looking out for one another in your social group is of paramount importance. I should have known, and in retrospect it is obvious.

Classroom role reversal is a method where I assigned topics to students 2 weeks in advance for an upcoming unit. It is a very high IDV method, and it failed horribly, but it is more relevant to teaching and therefore discussed in chapter 4 . 


\section{Chapter IV: Teaching Classes}

\section{Project Overview}

Teaching classes at St. Patrick's College (SPC) was a simple enough matter for me assuming the curriculum we developed was successful. Each of the three years of instruction were scheduled at 4 hours per week. This project obviously had a large amount of crossover with the curriculum development project. Given that my training methods with my counterpart were largely participatory, it also had a large crossover with the teacher training project as well. The biggest asset I had as a teacher at SPC was largely the result of three things being true simultaneously:

1. Being a relatively experienced teacher.

2. Having a core understanding of IT/CS.

3. Having taken the time to understand (as much as an outsider can) their culture and language.

As a result, the contributions I was able to make manifested in 4 specific ways:

First was that I was able to give better explanations of concepts in class because I had a deep understanding of them to start with. Second was that I was able to give better on-the-fly examples in class as a result of understanding their culture and speaking Bislama. As an example, in explaining the difference between RAM and Hard Drives, I came up with the metaphor of water tanks and water bottles. The water tank is the hard drive that holds everything. It is slow because you have to walk all the way to the tank to get some water. The water bottle is the small but fast RAM, where water is always available at your fingertips, but it does not (relatively) hold very much water/data. These cultural bridging examples and explanations were usually added to the curriculum materials after I made them up in class so that my counterpart could use them in future. 
Thirdly was the ability to teach faster when it was needed, which primarily comes from experience as a teacher before. After a few months teaching in Vanuatu, I was able to successfully check in with students more effectively throughout class and keep up a faster pace when needed. The benefit from this was primarily toward the end of the school year, when time was short to prepare students for exams. There isn't a sustainability aspect to it, but for those particular students, that ability helped them to be better prepared for their exams. Fourth and finally was the example of professionalism, good teaching practice, and (where appropriate) American teaching methods that I was able to provide. This is not to say that teachers at SPC were unprofessional or should adopt American teaching methods, but rather that the example I provided was there for other staff members and my counterpart to emulate if they saw fit.

\section{Challenges: Curriculum Choices}

The curriculum choices (as outlined in chapter 3) had implications on the challenges that my counterpart and I faced in teaching those curriculum methods. Among them were the difficulties of using a single teaching method, the challenges of integrating positive reinforcement, and the difficulty of removing shame from the learning environment. First is that if the teaching method is class discussion but your topic is Python syntax, there is no hope. Thus, choosing the right method (of those available from your own curriculum resources) is critical and not usually so obvious. For instance, there are many situations in which the same topic has two different sub-topics that are better explained with different teaching methods, like Python syntax with a PowerPoint but program design with class discussion or whiteboard notes. The second major drawback is 
that the teaching method you choose might not be available one day, for example if you have a PowerPoint ready to go and then there is no electricity.

There are also challenges to teaching mechanics that are sourced from curriculum decisions but based in Vanuatu's low IDV. For instance if a teaching method requires class participation, the mechanics of getting students to participate is often difficult. Another is the mechanics of keeping students from trying to memorize correct answers instead of understanding concepts and principles. A third is maintaining positive reinforcement in the classroom and eliminating shame. The IDV related challenges to teaching mechanics that result from introducing a high IDV curriculum are endless. As are the possible new approaches to meet those challenges.

\section{New Approaches: Curriculum Choices}

As a result of choosing only one teaching method from the available methods in the curriculum for a given unit, it is sometimes difficult to choose only one and the right one for the job. In such situations I found it was always most successful to just choose whichever worked best for both and accept the compromise. The temptation is to use both, but it will end in disaster. This is primarily because of low UAI, low LTO, and low MAS... known colloquially as island time. Each class period is only one hour long. Take into account passing time, incorrect (manually rung) bell times, and the time it takes for students to:

1. Remove their sandals ${ }^{19}$ at the computer lab door

2. Turn on the computer

3. Log in, etc

\footnotetext{
${ }^{19}$ In many south pacific cultures, this is normal and respectful, including Vanuatu. However, this was not normally practiced in SPC classrooms. We did it for the computer lab however because dirty feet and computers do not mix well.
} 
... and it is really only 45 minutes long at best. In fact, Fridays and special occasions at SPC are half days ${ }^{20}$ and utilize 45 minute periods to begin with, so really only 30 minute classes can be guaranteed for any given day/topic. There simply isn't enough time to cover two distinct topics or sub-topics with different teaching methods. There is barely enough time to cover two distinct topics or sub-topics with the same teaching method. The context switch takes consequential time for both the Ni-Vanuatu teacher and the student. This would be more than feasible in America, but time moves more slowly in Vanuatu and trying to speed it up will only alienate people. This was probably the hardest teaching mechanics lesson that I had to un-learn. From an American perspective it is perfectly normal to use 2,3 , and even 4 separate teaching methods sequentially or even simultaneously. The time it takes to context switch is inconsequential and the America's high UAI makes simultaneous methods somewhat easy. This is simply not the case for Vanuatu.

The second challenge resulting from curriculum choices is limited availability of certain teaching methods, most commonly not having power. The only approach that I learned was to adapt or die. It's the harsh reality. This means that (from the curriculum decisions we made) all topics have to be available in more than one teaching method and preferably at least one that is still functional given any combination (and sometimes all) of the following constraints:

1. No electricity

2. Shortened class

3. Widely spaced classes (Due to cancellations)

4. Broken computers

\footnotetext{
${ }^{20}$ As a further example of being a low LTO culture, Fridays are half days because that is payday and teachers want to get to the bank.
} 
5. Sharing computers

6. Long term student absences (Common due to travel time to/from school)

7. Too hot to concentrate inside

Note the difference between the first and second approaches to curriculum sourced challenges in teaching mechanics. The first is about what methods to use on a given day and only using one, the second is about using a method that works given uncontrollable constraints. It was quite common for them to combine and produce truly devastating results. For instance, upon my arrival at St. Patrick's College, the teacher's cabinet contained hand-drawn charts of instructions about how to use formulas in Microsoft Excel. These were not informational decorations as I assumed. My counterpart informed me that they were used as visual aids in teaching when there was no electricity. SPC's former Peace Corps volunteer had taught Microsoft Excel without electricity.

Perhaps more helpfully, the only new approach that I found was to develop a low UAI, low LTO, and low MAS for myself:

- Class is cancelled, you've covered half the topic, and term ends tomorrow: oh well, it can be picked up again when the next term starts. (low LTO)

- Power just cut and you're covering Python syntax: oh well, just use the whiteboard, extend the time it takes to teach it, and check the students understanding level more often. (low UAI)

- The grant application that you and the school admin committee have been working on for months has just been delayed another 5 months: oh well, it will get done when it gets done (low LTO) and if it doesn't get done then 
at least we all have had the experience of working together (low MAS) as a successful team.

These are all real examples of actual UAI, LTO, and MAS setbacks that I faced during my service.

\section{Challenges: IDV}

Regarding my teaching experiences in Vanuatu, Individualism (IDV) was by far the most important of Hofstede's Cultural Dimensions (HCDs) for me to understand. This holds true for me from both a professional and a personal perspective. It affects every level of Vanuatu society and culture in ways that I (as an American) could not understand until I lived there, even with 2 months of top-notch cultural training, fluency in the native language, and working very hard to integrate as Peace Corps expects. After completing my Peace Corps service, I still don't believe I will ever truly understand this cultural dimension fully. One might even be wise enough to expect this going in given the drastically opposite values of this dimension for the United States and Vanuatu ${ }^{21}, 91$ vs 14 respectively [5]. In fact, the United States has the highest IDV value of any nation on earth and Vanuatu has one of the lowest [5].

One way to explain this is that low IDV or 'collectivist' societies are ruled by consensus and view confrontation itself as a form of conflict. Thus confrontation is rarely (if ever) seen as a resolution to problems, but rather as an exaggeration of them. Collectivism is so high in Vanuatu's culture, that when teaching it is incredibly difficult to even get students to have an individual, original thought. This is not to say that $\mathrm{Ni}$ -

\footnotetext{
${ }^{21}$ Actual value is for Fiji.
} 
Vanuatu students are unintelligent, but that they follow a collectivist thought process. The algorithm that determines their thoughts is not complete without input of the opinions from those around them and those in their social and loyalty groups. For example, when teaching, students would constantly whisper to each other after I asked a question. In stark contrast, an American classroom is usually dead silent following a question because each student is individually thinking what their answer might be. Even rarer are critical thinking skills. When opinions have been dictated by the thoughts of the whole for one's entire life, critical thinking skills are never developed. This was painfully evident in higher level senior secondary classes where problems often require these skills just to interpret the question, rather less synthesize an answer. Most often these problems occurred on the student's final exams.

\section{New Approaches: IDV}

Of the many challenges to teaching mechanics that are sourced in curriculum decisions but based on high IDV methods; increasing positive reinforcement, removing shame, combating rote memorization, and increasing class participation were the most common for me. For positive reinforcement I used encouragement as much as I could, developing a nurture (over nature) side for probably the first time in my life. For removing shame, a simple (and strictly enforced) classroom environment policy did the trick. This most often cropped up from a student laughing at another student for having a wrong answer. The policy included the threat of detention and (with occasional reminders) this created a much more positive learning environment where being 'wrong' was not shameful. I also often gave quick talks about the importance of being wrong in the learning process. 
Increasing class participation and combating rote memorization were a bit tougher and never had simple fixes, but some methods that worked were:

- Repeated explanations of what plagiarism is and reminders to avoid it

- Utilizing participatory methods in class

- Outdoor class discussion with significant positive reinforcement

- Asking questions for which there is no correct answer

- Assuming the wrong answer to force students to correct me

In the classroom, I would often ask students for a vote. This forces them to choose, although they would usually look around to vote with the majority. The best method for promoting higher IDV without being adversarial was to use participatory methods. In a lab setting, computers are naturally individual-driven because only one person operates the mouse and keyboard. By making sure that only one student is at each station (which is difficult with a 2:1 student/computer ratio) it requires the students to not only think individually but also act individually. If there were not enough computers for a 1:1 ratio for any lesson that needed it, the lesson simply had to be taught multiple times with sections of the class. For this reason, I found it very successful to teach everything (even things like PowerPoint presentations) on their screens in class. Even if teaching things on their screens in class meant much more work for me in giving instructions over and over again, their learning was improved.

Among the more adversarial methods, I would sometimes ask students a question to which there is no correct answer, only an opinion, like: 'Which is more important, privacy or security?' Upon giving their answer I would make a counterpoint to whichever side they chose. Upon asking another student they would choose the opposite of the first, so I would make another counterpoint to that side. This pattern would repeat until the students realized the fundamental point and began to form their own opinions instead of 
searching for the correct answer via trial and error. Lastly I would often assume an incorrect statement and run with it until a (pre-planned) problem arose, for instance: "A string variable holds a number, so what are the possible values for it?" After giving seemingly endless answers that I would say are incorrect but not why, eventually a student would speak up and correct my initial assumption. I would ask who else thought that but didn't say anything and inevitably there were some every time. Slowly they learned to question the unquestionable. This is particularly difficult for them given both the low IDV and the high PDI of their culture. Challenging a higher authority with individual thought was very difficult. Given how adversarial some of these methods to challenge low IDV are, positive reinforcement (constantly) was required. Sometimes with extrinsic rewards like cookies and pencil-top erasers and sometimes with intrinsic ones like compliments and attention.

These gains in IDV and critical thinking were hard fought but worth it, because their final exams are written by educated individuals who assume the presence of this skill. For example: a final exam question for year 13 was: "Explain the link between ICT and Climate Change. Give examples and justify your answers. ${ }^{22,}$ Despite that this is not a required topic or idea in the curriculum, the students (in their final exams) were required to link the two and explain the road that connects them. No amount of rote memorization can adequately prepare students for these kinds of questions.

\footnotetext{
${ }^{22}$ One possible correct answer: ICT requires electricity $->$ electricity must be generated $->$ generated electricity is mostly from non-renewable sources $->$ non-renewable electricity generation contributes to greenhouse gas emissions $->$ greenhouse gas emissions directly contribute to climate change. Examples would include: The SPC computer lab $->$ the SPC generator $->$ Diesel Fuel is used $->$ The SPC generator outputs smoke -> Smoke contains CO2 -> CO2 contributes to the greenhouse effect which warms the planet
} 


\section{Challenges: PDI}

Power Distance (PDI) is somewhat easier to understand than IDV. The classic example is how comfortable a person is talking to their boss about feedback and how comfortable that boss might be receiving such feedback. The value for the United States is a moderate 40 and for Vanuatu ${ }^{23}$ it is 78 [5]. This high PDI value indicates that authority structures in Vanuatu are to be respected at nearly all costs. Even if principals, chiefs, members of parliament, and grandparents are acting foolishly, their decisions and words are to be respected and even revered because they are in positions of power. For teaching, this is naturally most evident in classroom environments. Most students view speaking in class as the height of inappropriate behavior and will only very quietly (and terrifyingly) respond to direct questions after much prompting. This phenomenon is even further exaggerated for me for two reasons, the first being that I exhibit a lower PDI than typical Americans and the second being that I have a somewhat intimidating presence and personality to $\mathrm{Ni}-$ Vanuatu students as a $200+$ pound 6' 3" tall white man.

\section{New Approaches: PDI}

The Power Distance Index (PDI) is very important to understand in Vanuatu, and particularly at a formally structured institution like SPC. With such a high power distance, seemingly innocuous things can cause big problems, in the classroom, out of the classroom, as a staff member, as a community member, as a white man...everywhere. There are two things one can do, violate it or work around it. Deciding when, where, and how is the important part. Generally speaking, violating it when you are the powerful is

\footnotetext{
${ }^{23}$ Actual value is for Fiji.
} 
ok so long as steps are taken to make sure your methods are still effective and respecting it when you are the powerless is the only effective and/or appropriate route.

In the classroom, the high PDI of Vanuatu (among other factors) creates an environment in which students will not speak during class. This is problematic given that research shows over and over again that participatory methods are more effective [15]. Because of this, my counterpart and I had decided to use such participatory methods. One approach that I tried was to reverse the classroom roles, where the students had to give presentations about a topic that they had researched in advance of the rest of the class. I tried this with the year 13 students $^{24}$ and the Software Ethics topic. In retrospect, such a theoretical and difficult concept to grasp was not a good choice. Regardless, it was marginally successful from a PDI perspective. Students did become more comfortable with a low PDI but it created two other problems. First is that the other students did not learn the material very well and second was that (upon me re-teaching the material) the student that originally taught the material felt like they had failed. I judged that the PDI benefit was outweighed by the IDV (shame) cost and didn't use this method again.

Getting feedback is also extremely difficult in the classroom. Students are terrified of giving (especially negative) feedback. For instance I asked the students to tell me (by interrupting me) if I used a word they did not understand, like ambiguous. They literally laughed at me. They thought I was making a joke and naturally in a high PDI culture they are supposed to laugh regardless of whether or not it is funny. My counterpart then explained to me that they will never interrupt me. We compromised that

\footnotetext{
${ }^{24}$ I generally tried new teaching methods out on year 13 students because it was the smallest (6 students) class, most mature, and most exposed to the methods of international teachers.
} 
they would raise their hands instead, which they never did. In fact, raising hands even just to participate in class was such a challenge that I sometimes made all students hold their hands up until they had answered a question or made a comment. This worked but given the extreme nature of it, it was a last resort.

I didn't let the high PDI of Vanuatu bother me and I didn't work to change it at all. This is because there is little need for anything but a high PDI in post-secondary education or any career they might pursue in Vanuatu. The same is true for participatory methods. While I used them in class, this was to further their learning and raise their IDV, which they definitely do need in post-secondary education and careers. This is due to the very different culture of those they are competing with for positions and opportunities in the capital city. This is the kind of experience that most Ni-Vanuatu teachers would not be able to impart on students because most Ni-Vanuatu teachers would not understand the culture of CS/IT companies and roles. Even those in the capital city, for instance, what working for a cell phone company in the capital might be like.

\section{Challenges: MAS}

Next to discuss is Masculinity (MAS) which is a bit of a misnomer. This is because Hofstede began his work into his Cultural Dimensions Theory in the late 1960s, when 'masculinity' was still synonymous with achievement and accomplishment and femininity was not. Regardless, the United States of course has a relatively high value of 62 compared to Vanuatu's ${ }^{25}$ value of 46 [5]. This difference creates a lack of motivation in students despite that the entire purpose of being in school is to accomplish an

\footnotetext{
${ }^{25}$ Actual value is for Fiji.
} 
education and higher intellect. At a prestigious secondary school it is less of a problem than elsewhere, but it was still very common for students to just not care about getting assignments in, learning concepts, attendance, etc. I would surmise that this is because being in school and getting an education in Vanuatu is very widely seen as important, but accomplishing the goals of schooling (education and intellect) is not. I would also surmise that this (seemingly strange) split is due to parents not being educated themselves. Thus, going to school is the goal in their minds. In addition, one must also keep perspective regarding the parent level of involvement, information, and education. They get a single progress report per year in English, which they usually can't read. This means that even though some parents might prioritize in-school accomplishment for their kids, they have little means of enforcing that accomplishment requirement on their kids.

\section{New Approaches: MAS}

The most common approach that I took to raising MAS was centered around role models. I used assignments like researching the founder of Telecom Vanuatu Limited, a mobile carrier. Role models allow students to see who they can become and what they can accomplish, which is directly related to MAS. The most effective approaches for this were more commonly outside the classroom however, as discussed in chapter 7.

\section{Challenges: Language and IT Ethnocentrism}

Perhaps one of the biggest barriers for most internationally placed teachers is language. Luckily, Peace Corps Vanuatu has an excellent language training program. Also, Bislama was not a difficult language to learn, my students spoke English, and my 
classes were to be taught ${ }^{26}$ in English. One might, at first glance, think that this solves all communication problems. This would be a woefully incorrect assumption. The lack of a language barrier does not imply the lack of a communication barrier. One of many reasons for this is that my English is naturally much more diverse in vocabulary, complex in grammar, and faster in speech than typical SPC students are comfortable with. Another is the cognitive delay added when one party in any communication is not using their native language. In this case, my students were forced to hear $->$ translate $->$ process $->$ think $->$ translate $->$ and finally answer back. This cognitive delay can often appear as misunderstanding, when really it is just a delay.

Last but probably the most important is the difficulty of ethnocentrism in IT/CS education. For starters, most native English speakers don't even realize it but the biggest barrier to learning IT is the internet. The internet's massive ethnocentric assumption is its language. Over $55 \%$ of the internet is in English, and the vast majority of the other $45 \%$ is in other various major world languages [9]. Thus, for students at SPC who only speak/read/write middling proficiency English as their $3^{\text {rd }}$ or $4^{\text {th }}$ language, this represents yet one more barrier. As a sort of double blow, language is both a cultural and IT-specific barrier, given that students at SPC would have no hope of understanding either "ambiguous" or "toolbar" without further explanation. Other ethnocentric assumptions like the save icon in Microsoft Word are difficult to explain and take more time in class. If one has no idea what a floppy drive is, the save button makes zero sense.

\footnotetext{
${ }^{26}$ St. Patrick's College is an English immersion campus. Technically students are not allowed to speak Bislama without risking detention.
} 


\section{New Approaches: Language and IT Ethnocentrism}

The approaches to these problems are somewhat simple to think about and somewhat difficult to remember to do. First and foremost is to use simple English. Also necessary was to repeat explanations, vary explanations, and check in with students to make sure they understand while teaching. The hard part is remembering to do all of this when you have been speaking English as your native language for your entire life.

There is a quote from Nelson Mandela that explains this next approach very well that also comes up in Peace Corps training: "If you talk to a man in a language he understands, that goes to his head. If you talk to him in his language, that goes to his heart" [10]. Sometimes, what you are trying to convey as a teacher is just too complex or a student is so confused that being able to leave the English immersion environment for a few moments will truly make all the difference in the world for that student's understanding. Being native level fluent in Bislama helped me and my students in exactly such situations, where learning the concept is more important than understanding it in English. In such situations it is critical to recognize that lowering the frustration level of the student is more important than any possible learning value from a few extra moments in English.

The last challenge in language, that of the cognitive delay, is probably the hardest to remember to constantly be aware of. Americans can stand about 7 seconds of silence following a question before they have to say something. If this sounds unreasonable, just try it in a conversation at some point. At around the 7 second mark is when the discomfort will be almost tangible. This is about how long an American teacher waits 
before repeating a question or moving on. However, due firstly to the cognitive delay, secondly to the lack of critical thinking skills, and thirdly to the time it takes to build confidence enough to answer, that time for Ni-Vanuatu students is about 45 seconds. This seems like a lot, but after 2 school years, countless workshops, and many classrooms in Vanuatu, I am quite certain, and it is a very difficult behavior to get used to in teaching. In fact, this is so ingrained that I often used an actual clock to time myself before speaking again in class.

Challenges to the ethnocentric nature (or American nature really) of IT can be pretty difficult. How does one explain the concepts of privacy and piracy, rather less the difference between these easily confused and similar sounding words to people who grew up without either? ${ }^{27}$ The only way to do so is very carefully and with really well thought out examples. For privacy, I used a very contrived example of the cultural practice of creeping $^{28}$ and Facebook or text messages between the two individuals involved. For piracy, I used the original meaning (ships and theft and rum) and simply explained the new meaning. However teaching this concept after intellectual property/patents/trademark/copyright, was critical. This was a prerequisite knowledge gap as discussed in chapter 3 .

Finally, in such a high PDI culture, getting students to tell you when you are using language they don't understand or making ethnocentric assumptions, in my experience, is

\footnotetext{
${ }^{27}$ Technically, piracy is legal in Vanuatu and all software (even that of the entire government) is stolen. The primary provider of computers (Computer World) uses unlicensed copies of Windows software on all of the machines they sell.

${ }^{28}$ Creeping is a (usually) teenage dating ritual in which boys "creep" on girls at their homes at night to express their interest. This is in a culture that would otherwise never allow their social interaction in the literal light of day. The practice is slowly dying with the adoption and widespread use of mobile phones and texting. In Peace Corps training, we underwent a creeping simulation because this is a normal and culturally appropriate action in Vanuatu. Responding with anger or violence is inappropriate.
} 
impossible. It also isn't their job. Thus, as a teacher I had to learn to catch myself when I made these mistakes, which simply took practice. 


\section{Chapter V: SPC Staff Capacity Building}

\section{The Project}

During my two years at St. Patrick's College, I worked with many different staff members on many different projects. This ranged from training my teaching counterpart to communication-style workshops to computer training for Administration. Two of the ten core expectations of Peace Corps volunteers are to share skills if, where, and when needed as well as to be 'on the clock' $24 / 7$ as an unofficial ambassador of the United States. This meant that part of my job was to help with things that weren't in my job description. Sometimes that meant helping a mama better understand why Americans aren't all rich and sometimes it meant teaching the principal how to make a budget. I am by no means an expert in such topics, but the entire theory of Peace Corps is that many of the skills (but not a volunteer's primary project; CS/IT Education in my case) so desperately needed in these communities don't need an expert. They just need someone who has taken the time to integrate, learn the language and culture, and can be effective on the ground level at what the development sector somewhat nebulously calls capacity building. Capacity building usually just refers to the training of skills or processes into people or institutions that improve their capacity to learn, live, and work.

One of the capacity building projects that I worked on was with the Academic Principal, Kathy Tahi. She and I worked together to automate scheduling and remove inefficiencies using a free program called Free Educational Timetabling (FET) [16]. One such inefficiency was having double periods for all classes, when really only a few (lab science) classes needed and/or wanted them. I worked to achieve this from the very 
beginning because I recognized the difficulty in making lesson plans for classes that are either 1 or 2 hours long. This is especially true in Vanuatu with its low Uncertainty Avoidance Index (UAI) and low Long-Term Orientation (LTO) values. These low values mean that classes were often cancelled without notice and frequently many times in a row. Thus, having uniform class lengths made recovering from such cancellations much easier for everyone. We also added things like committee and department meetings as well as study hall. This allowed for better scheduling, attendance, and reliability of meetings as well as removing the free periods students used to waste time and be disruptive. Without FET, such things wouldn't have been possible. Even with it, the SPC schedule is so enormous and complex with such limited resources (classrooms and teachers) that the program often took $30+$ seconds to find a valid combination.

Another such project was with the Deputy Principal during my second year, Elwin Boi. The project didn’t really get anywhere while I was still at SPC, but we started the idea for it and teachers seemed to like it. Essentially the problem was that the detention and discipline system wasn't working because everyone was being disciplined all the time. Also, detention was manual labor in the school farm for hours on end. This sounds horrible to Americans, but to Ni-Vanuatu students that sounds like any day that ends in y. Elwin and I designed a system based on positive reinforcement with the loss of privileges for students instead of imposing punishments. At the time that I left, the staff were really excited about it. Given the complicated administration structure of the Anglican Church's Education Board, it will probably be a long time (if ever) before it is implemented. 
In Peace Corps terminology my 'counterpart' was Newman Tahi, with whom I did the vast majority of my capacity building work by training him as my own replacement. During the first school year (Feb-Nov) of my service, we had two sections of year 11. This enabled me to teach the course first and for him to simply copy my teaching methods for the following section. All of the courses (11A, 11B, 12, and 13) met for 4 hours per week in the form of two 1-hour classes and one 2-hour double period. For the one year 12 section, we team-taught this course so that he could become confident teaching on his own. Finally, in year 13, he was a student as I taught the course. Newman Tahi only had a hardware certificate from the Vanuatu Institute of Technology and thus he needed time to learn the (relatively) advanced topics of year 13 thoroughly before attempting to teach them.

During my second year of service, Newman taught year 11, 12, and 13 on his own. The entire school (except lab sciences) had also switched to 4 1-hour classes per week. He taught all levels at my insistence and his reluctance. He was not confident in his computer knowledge or English level. As a compromise, I agreed to always be in the classroom and willing to take over or help out so long as we had prepared for the class session beforehand. This was because preparing for class sessions is critically important in teaching but is a somewhat new concept to teachers in Vanuatu, and thus required constant reinforcement. Generally speaking, my methods for training Newman were entirely participatory. The students were aware that I was training him and that sometimes I would (as discreetly as possible) correct something he was doing mid-class. A big help here was that Vanuatu has a lot of non-verbal and body language. 
Overall Newman exceeded all expectations that anyone could have had for him regarding his newfound teaching abilities. Starting his training process with SPC's former volunteer in 2012 with only a hardware certificate, by the end of 2015 he was teaching year 11 and 12 Computer Studies. He is also capable of teaching the majority of the year 13 ICT curriculum, although given the difficulty of its topics, I doubt he will ever be able to teach it fully or confidently without significantly more training. It is important to note that these are not just the opinions of me, his biggest supporter. The exam results of his students in 2014 and 2015 were only slightly below the SPC teacher's average ${ }^{29}$.

Essentially this was a huge success for a few reasons:

1. First is that there is no education program to become certified to teach these prescriptions in Vanuatu,

2. Second is that every other senior secondary teacher at the school was required to have a bachelors or higher in their subject, and

3. Third was that he out-performed some of these more highly qualified teachers at the school.

\section{Teaching/Curriculum Crossover}

Naturally with regard to training my counterpart and other SPC staff, some of the challenges that we faced were the same challenges to developing curricula and teaching mechanics. As Newman had never been a formal teacher before, it was doubly hard to train him how to teach a subject as foreign as IT/CS as well as the basics of teaching mechanics, for instance how to run a classroom or building student relationships. However, one of the biggest factors that helped with this was that Newman had a very flexible and laid back personality and was also somewhat exposed to many of the intercultural differences between us, having trained with the former SPC volunteer for 2

\footnotetext{
${ }^{29}$ For 2014 , he was $4 \%$ below the SPC average and for 2015 he was $11 \%$ below SPC average. This was to be expected given the more difficult concepts and teaching all three levels.
} 
years previously. What remaining challenges that crossed over from teaching and curriculum development had similarly successful approaches applied.

\section{Challenges: MAS and LTO}

The primary challenges that cropped up around Masculinity (MAS) and Long Term Orientation (LTO) were in training my primary counterpart, Newman Tahi. Vanuatu has a low MAS, which implies that accomplishing things for oneself is less important than social structures and family ties. This was very evident to me in my counterpart. He wanted to become a trained teacher and know computers and he liked teaching, but the same difficulty with transforming ambitions into actions that was common in my students was also present in him. This often presented in an inability to accomplish tasks independently, which is of course a crossover from being a low IDV culture. Another example might be the prioritization of drinking Kava at night over doing work. This is a widespread phenomenon in Vanuatu, so much so that men are often stereotyped for wasting every cent they earn drinking Kava to avoid their families. This prioritization of Kava in one's social group over working independently is also a strong example of low LTO. The social group tonight was more important than the lesson plan for next week or even tomorrow.

\section{New Approaches: MAS and LTO}

In training my counterpart and other staff members, the obvious result of low MAS and LTO does not exist: consequences. In a student environment, if a student prioritizes playing a game or swimming in the solwata ${ }^{30}$ over their homework, there are

\footnotetext{
${ }^{30}$ Solwata -> salt water -> ocean
} 
consequences the next day. Be it detention or a stern talk from a teacher, there are consequences. This is not the case with staff at SPC, in nearly any regard. In a nonconfrontational culture, consequences are rarely if ever direct, never severe, and usually ignored. Even when they do happen it is often not in a cause -> effect pattern, but rather an unknown causes -> small effect trend. As an example, if a teacher did not show up to or properly plan for class, the lesson would be terrible. And no one would care. An absence might get recorded and after far too many of them, there might be a warning. In a culture without consequences the only approach that I found that worked was, unfortunately, shame. This is (obviously) because shaming people is already such a huge part of how their culture functions. I tried very hard to avoid it because it goes against every fiber of my being, but sometimes I decided to utilize it and pick up the pieces afterward. For instance, after my counterpart failed to prepare for class with me at a designated time 4 times in a row, I showed up to class as always but did not guide him or take over as I usually did. We had not prepared and this was the consequence. It was cruel and painful for me to do, but I allowed him to crash and burn in front of the entire class when he did not know the material or have a lesson plan. The important parts to this approach are that it is a very occasional last resort and that a conscious effort is made to pick up the pieces afterward. This is accomplished of course, through the opposite of shame: positive reinforcement.

\section{Challenges: PDI and Privilege}

Outside of the classroom, high PDI is arguably most important HCD to work within and around. Not only respect, but even reverence sometimes, for authority is expected. This is particularly true for those who hold religious authority positions, like 
the school chaplain. As an example of this, I regularly ended up in conflict with the principal and original deputy principal ${ }^{31}$ of my school. This was not because they did not like what I was saying or doing or how I was saying or doing it. It was because I was saying or doing it. In many cases, I (and other equally ignorant international teachers) would speak up about an issue when it was not our place to do so. During my first year of service for instance, the deputy principal of the school was a very "stronghed ${ }^{32,}$ individual. One night, in the middle of term 3 and 8 months into the school year, he ordered that the computer lab be shut down because no one was monitoring it. Seeing as I had run the lab like this for many months with student captains monitoring the lab at night, I was naturally confused. I went to speak with him about it and the conversation blew up in to a full blown Peace Corps mediated security incident. I had violated the high PDI of their culture and a series of other factors caused this (otherwise minor offence) to blow up. Of course, other things also constitute such violations. For instance, giving up the front seat of the school truck for the principal was implicitly required, as was calling him principal. In fact referring to all individuals in Vanuatu by their title is customary and respectful. It took my own students many months to become comfortable calling me Tim, and many never did. They insisted on Mr. Tim or Teacher. Unfortunately I did not learn enough of these lessons quickly enough before damage was done and because confrontation is avoided at all costs, no one told or even intimated that there might be a slight problem with the things I was doing and/or saying. Luckily this did not extend to my counterpart, partly because he is a very laid back person, and also because our power

\footnotetext{
${ }^{31}$ The deputy principal for my second year of service was an excellent person to work with.

${ }^{32}$ Translation: "stronghed" -> strong head -> hard headed -> stubborn/arrogant/domineering, etc
} 
level was the same. If anything mine was higher as I was technically the department head over all elective courses ${ }^{33}$.

Along these same lines comes the concept of privilege. There are other factors that provide it in my case, but none so much as being a white and educated man. It afforded me a great deal of privilege too. It caused challenges like the one I discussed in chapter 3 regarding the new year 10 IT/CS classes. The administration of the school never told me that it was a (huge) problem because they didn't want to disappoint their new Peace Corps volunteer. If I had wanted to, there were many things I could have done that no other staff member could have. This, at first, sounds like an upside, but in my experience it landed me in trouble more often than it benefited me. For instance I could type my own exams quite easily and I knew precisely how to use the copy machine in the main office. The staff at large could not do either and the office secretary always did it for everyone. Even the office secretary didn't type that fast or know how to do multi page copies or duplexing. I later taught her both skills, but nonetheless it was seen as quite rude when I once jumped a long line that I didn't know existed after the secretary went to the restroom. I accomplished the same copying task in 30 seconds that it took the person whose full time job it was $10+$ minutes. Then I walked out and upon returning to the staff room received some strange looks. It never even crossed my mind that anyone was waiting for their copies, that the copying was a very slow process for everyone else, or that teachers might not know how to use the machine themselves. I should note that there

\footnotetext{
${ }^{33}$ This included French and Agriculture: two subjects for which the sum total of my knowledge could fit on a postage stamp.
} 
is also an element of privilege in that I have no fear of said copy machine, because I know how to use it already and because I could afford to replace it if I broke it.

\section{New Approaches: PDI and Privilege}

The only culturally appropriate (and therefore effective) approach to PDI challenges outside of the classroom was to work within it. Similar to how my students needed to grow a high IDV for my classroom environment, I needed to grow a high PDI for their cultural environment. An example of this to better illustrate what it might look like might be opening all meetings with a prayer followed by thanks for everyone. This shows respect for everyone who had even the slightest role in planning or executing the meeting and also thanks everyone for showing up, even if they are required to be there for their jobs. Another could be not reporting (even serious) misconduct of a principal. It is not appropriate. If and when feedback is needed, it will be asked for, which is basically never. A cultural example might be that a chief's decision is law, literally. It doesn't matter if almost every person in the community disagrees, the only appropriate way to 'fight' his decision is to quietly talk to other chiefs and hope they decide to help you.

Being aware of and constantly conscious of my own privilege was vital to integrating and being successful. As an example, getting the truth out of people is very difficult if they see you as a superior, meaning I had to work extra hard to be seen as an equal. I would wash my own clothes despite that men rarely (if ever) did this hard and intensive labor. The same was true for cooking my own food as often as was appropriate. Outside of gender roles, I would avoid speaking at staff meetings because meetings are an exercise in top-down order delivery, not conversation and decision making. 
Discussion was appropriate in small groups (most successfully one on one) before and after the meeting or at night while drinking Kava and only with those at similar power level as you. This is true both for situations in which you are the powerful and in which you are powerless. It is slower and requires a lot of redundant conversations with many individuals, but it is the only appropriate and successful method. 


\section{Chapter VI: Computer Lab Project}

\section{The Project}

The computer lab project at SPC was simple enough on the surface. To drastically improve or get an entirely new computer lab. We endeavored to create a new physical space, get new computers, a new server, a new power source, etc. The problems? Never ending. The solutions? Really hard. Of the challenges specific to the computer lab project, the primary ones were logistics, power, environment, money, software, and internet accessibility. Many of these are related to each other and have layers of subproblems, but each has distinct challenges and approaches that can be successful.

\section{Challenges: Logistics, Electricity, Environment, Money}

The most obvious challenges upon arriving in Vanuatu and more specifically at SPC are logistics, electricity, environment, and money. These are common problems in the developing world but the ethnocentrism of never having to deal with them (to the degree that one must in the Vanuatu) makes them quite a shock on first arrival. For instance, upon arriving at the school for my site visit (one week, mid training) I discovered that the satellite internet for which the Anglican Church pays dearly, had not worked in months and nobody knew why. It turned out to be an ethernet cable that had been chewed through by a rat that had once connected the satellite dish to the modem. Fixing this should be simple, except that the cable is over 50 feet long and the only place you can buy ethernet cables of any length is the capital city, which is a $\sim \$ 320$ round trip flight. Luckily the school had a splicing tool ${ }^{34}$ so I simply spliced the cable back together

\footnotetext{
${ }^{34}$ The former Peace Corps Volunteer, Melissa Burns, had left it in the computer lab.
} 
without the damaged portion. Unfortunately, the solutions to these problems are usually not so simple.

Kind of a broad term, logistics refers to everything that can (and will) go wrong but is not distinctly part of environment, money, or electricity. Some examples might be shipping times, using flights or boats or trucks (of which each have drawbacks and advantages) or even things as simple as making sure the school driver knows how to handle a box of computer components that just arrived on the plane.

At St. Patrick's College, stable electricity was very difficult to maintain. The school had a very large budget, but due to administrative mismanagement of funds and repairs, as well as a lack of any contingency planning, the generator was always breaking or the school was running out of fuel. Not only is generator fuel expensive it is also very dirty power. Poor SIN waves create an extreme burden on power supply units, which in turn break faster. In addition, due to low LTO and low UAI, the generator would always be turned on or shut off close to when it should be but really whenever is most convenient for any number of unpredictable reasons. Unannounced variations by as much as 30 minutes to half days were quite common, resulting in lost class time and computers being regularly subjected to instantaneous power loss.

The environment for the computers is also very challenging. In addition to being very hot and humid, there are also dust and insect concerns. The extremely large wasps for instance love to build their mud homes inside the computer towers because they are even warmer than outside. This often shorts out circuits and creates a hazardous situation in cleaning them out. The extreme expense of air conditioning makes it prohibitively 
expensive at around \$13 USD per hour. The alarming frequency with which the country experiences natural disasters is also an environmental concern. Plans must be made for dealing with earthquakes which can shake computers off of tables, tsunamis which can flood labs and cause power surges from poorly maintained generator/electrical systems, and cyclones which can cause the same problems as tsunamis. Cyclones also get the added bonus of high wind, meaning my $2^{\text {nd }}$ floor lab could (and did) flood. As if that wasn't enough to deal with, there are also volcanic eruptions which can cause huge amounts of dust and ash to fill the air, eventually clogging up fans leading to overheating units and shorting out boards.

Last is money. Computers are expensive not only to buy but arguably even more so to maintain. They break all the time and there is no ability to maintain money to the degree that they can be replaced when this happens. Getting money is relatively easy but getting easy money is very hard because it always comes with strings attached. In addition to the good strings like sustainability plans and compulsory trainings, often the strings are related to the goals of the money, meaning the goals of the (relatively) rich people and nations that donated the money. Unfortunately in my experience, these goals are rarely in line with the goals of the people of Vanuatu, they are rarely sustainable, and sometimes they are downright ill conceived. For instance, the grant program that I used to fund SPCs new computer lab had its primary goal as achieving a Universal Access Policy (UAP) of $97 \%$ geographically at $36 \mathrm{Mbps}$ or greater with $1 \%$ or less downtime. This is beyond unrealistic. In Vanuatu's terrain, a cell tower can usually only cover 4-5 kilometers at most. For context, the United States using the same speed parameter (with far more resources and significantly less difficult terrain) is at less than 5\% geographic 
coverage wirelessly and less than $20 \%$ via landlines [18]. In addition, the United States average landline speed is $11.7 \mathrm{Mbps}$ [12]. The grant was developed in Australia, by politicians that want a sound byte, which was precisely the source of some other major problems, like the re-organization of the entire program after accepting all of the submissions. More generally speaking, money always came with strings, which were usually ill-conceived.

\section{New Approaches: Logistics, Electricity, Environment, Money}

The approach that I found worked best for dealing with difficult logistics in Vanuatu: don't. That doesn't mean I let my computer shipment sit for 3 days on the sand at the beach after they were delivered, just that it wasn't my fault. The key here is that I was never, ever, ever, going to understand logistics or how to run them in Vanuatu anywhere near as well as the locals and by trying I would just look like a fool. What I could do was work with the locals so that they understood the specific-to-IT limitations of logistics. For instance, when the school had a Digicel representative come out to assess viability for $3 \mathrm{G}$ internet, I told everyone that the technician would need to climb up to the top-most point of the hill so that he could establish whether or not we have line of site with the tower. There was no way to do this when I mentioned it. I had no idea how to clear a path up a muddy mountain that looked more vertical than horizontal to me, but they figured it out just fine. It didn't even require adults. The year 12 boys dorm did it in less than an hour. As an even more hilarious story of just letting the locals do best what they obviously know better: when I was in the training village on day 2, my 4 year old host brother Stanley was watching me struggle for 20 minutes to open a green coconut 
with a busnaef ${ }^{35}$ and giggling. Right as I was starting to make progress, he walked right over and without a word (neither of us could speak Bislama yet) he took the 2 foot long sharp busnaef from me and in less than a minute had it open with a perfect drinking hole. Four years old and he was showing me up with a 2 foot long knife. I had inadvertently explained via miming what I needed and he obliged far better than I could. This was the only successful approach to logistics that I ever discovered. Teach them what is needed and let them do it. Simple $=$ Successful.

There is little hope of gaining electricity without external money. The same is true for computers, a quality space for the computers, and the logistics of purchasing, shipping, installing, and maintaining computers, power systems, and their physical spaces. Essentially most of the physical and logistical concerns boil down to money, so I was forced to figure out a way to either work around that need, or fulfill it. Fulfilling it seemed like a feasible possibility given that the Peace Corps had grant programs and the school itself was well funded. After some failed attempts through the school and the Anglican Church, I ended up using a grant from the Australian government. This program was specifically targeted toward Peace Corps occupied schools. While this was an excellent choice for the program, there were many challenges rooted in the fact that it was a top-down development program. For instance, program administrators did not understand that a complex Linux server running two nested virtual machines would not be sustainable in Vanuatu. The program was also designed with a clear misunderstanding of Vanuatu's basic power generation challenges as it relates to cost. (i.e. - generator fuel is expensive) Essentially, the lesson that I learned over time during my service was that

\footnotetext{
${ }^{35}$ Busnaef $=$ Bush Knife $=$ Machete
} 
with top-down development programs comes money, but also strings attached.

Unfortunately these strings are not just the hoop-jumping kind. Careful consideration and planning must be undertaken to minimize the damage. Most importantly, that minimization of damage should be done by locals who have been trained in the process, thus making the overall process and effect sustainable.

I was able to teach the staff at SPC about the grant process and how to build sustainable and well-designed projects so that they can utilize money from these programs smartly. These skills that I tried to impart are definitely proving useful given what eventually happened. The grant that my counterpart and I had applied for in month 4 of my service had still not been installed by the end of my service. This was due to never ending delays in the program, a cyclone hitting the nation's capital, and poor management of the implementation process. However, about 2 months after I left, because I had taught the staff at SPC how the process works, how to do a sustainability plan, how to build a cost model, how to plan for the unexpected, etc ... the computer lab is now installed and operating correctly. It is one of the only installations in Vanuatu from this same program that does not have serious sustainability and functionality problems.

Protecting computers from the physical environment is very challenging even for everyday things like insects, heat, humidity, dust, rain, rats, and people. It requires a secure room to keep unauthorized people out, as well as 4 layers of different materials on the windows to keep various things out. Chain link fencing keeps people out, 1 inch further in the glass keeps water and wind out but needs to be capable of opening during 
lab use for airflow. One inch further in from that must be chicken wire to keeps rats out, and one final inch further in from that must be screen material to keep insects out. It has to be in this order and spaced apart or rats will destroy the screen or some kid will destroy the glass, etc. The only approach to the heat and humidity was to keep airflow moving in the computers and in the lab itself with fans and open windows.

There is absolutely nothing that can be done to safeguard against mother nature. The buildings and structures in Vanuatu are generally not capable of withstanding these forces. St. Patrick's College, is again, the exception. As an entirely concrete school it will withstand most natural disasters. However the windows are the primary point of weakness in any computer lab due to the need for windows that open. This necessitates that the windows be Louvre style since sliding glass is prohibitively expensive.

Unfortunately Louvre windows have zero ability to stand up to powerful storms and rain.

\section{Challenges: Software and Internet}

Challenges to software at SPC were numerous. Among them were:

- Inability to update application and/or system software

- Large updates take forever to download

- Inability to research issues during installation/operation

- Windows Automatic Update breaks N-Computing

- Software that required internet connection (SaaS) not possible

These challenges created serious implications for how the computer lab was run, for instance, security software was very difficult to run due to the constant updates. The $\mathrm{N}$-Computing system is a method of virtualizing one tower into 6-10 workstations.

Unfortunately, Windows updates broke this system constantly and Windows has a nasty habit of installing updates that turn on automatic updates. Then, not only would those 
automatic updates usually break N-Computing, they would also tie up the internet. Software that required the internet to function, like the latest versions of the Adobe creative suite, were completely impossible. Even minor application bugs that could not be fixed became unbearable as a result, particularly without the internet to research and/or fix a potential installation or operation problem.

\section{New Approaches: Software and Internet}

The approach that I found worked best for software was planning ahead. A lot of planning ahead. Similar to the planning required in order to develop curriculum in the capital with access to internet and a comfortable environment, so too is the amount of planning (if not more so) involved with maintaining the computer lab's software. I usually kept a running list of things that I needed to download in the capital, then placed them all on 2 different flash drives (in case one got infected with the recycler virus ${ }^{36}$ ) to take back to the island with me. Performing lookaheads on the curriculum units to try and anticipate what software might need to be downloaded or updated was also extremely helpful, but again, dependent on getting to the capital. A sustainable approach to software would have unfortunately required internet while I was there. Fortunately, reliable and fast internet was installed for the first time at SPC in Nov 2015 with the same grant program that provided for the computer lab.

\footnotetext{
${ }^{36}$ A virus popular in the US in 2005 that takes all files on an unprotected drive and moves them to hidden files with shortcuts or deletes them entirely.
} 


\section{Chapter VII: Gender and Development Project}

\section{The Project}

Most Peace Corps countries have a Gender and Development (GAD) committee of volunteers that work on project planning/implementation and teaching tools that are sensitive to the gender context of that specific country. For Vanuatu, I was on this committee and a great deal of my work on it directly influenced the new approaches that I implemented in the classroom, with my school's staff, and as a volunteer overall. Among these approaches included the challenging (within limits) of gender roles, teaching life/leadership skills, and instilling the ideals of adult reproductive health and healthy relationships. I also learned some valuable information about how teaching adults is different from teaching kids in Vanuatu. Many of these lessons translated directly into the classroom and others translated more into my role as an on campus mentor for students.

Moving the definition of gender roles was a large part of my work on the GAD committee and it crossed over directly into my classroom and my work as a staff member as SPC. In the classroom I did things like pairing up students as boy-girl combinations. In fact for one assignment I had students make a music video with their partner singing for them. I also gave them assignments that were related to role models, which is an entirely new concept to most students at SPC. For example the year 11 students, while practicing typing, were required to do a one page essay about an opposite gender role model that they admire and why. Most chose people like Rhianna, Beyonce, Zac Efron, and Justin Bieber, but that's a different problem. In fact, it is a problem that one of the GAD exercises addressed directly, successfully, and with participatory methods. It is called Taf 
Tumas $^{37}$ and essentially the exercise has participants define what they like in certain people like Jackie Chan and Taylor Swift and then define who is more 'Taf': Jackie Chan or Barrack Obama. The trick is that, at the end, it is revealed (every time) that they like the qualities of roles models more despite wanting to be like Hollywood stars. The lesson usually hits home that the people that they really want to be like are not the same people that they think they want to be like. These aren't decisions we push on them, these are their own judgments. We just provide the question/comparison framework and facilitate the session in Bislama.

As a staff member, I tried very hard to work with the female members of staff to build their capacity as more successful teachers, administrators, and community leaders. For instance I worked with the Academic Principal to teach her how to use Free Educational Timetabling (FET) to automatically organize the schedule of classes each term. This saves her two weeks of tedious work every term and improves the schedule's efficiency on top of making her skills one of the most competitive in the country for her job role. I also required that my year 11 students entering Computer Studies be the top 11 girls and top 11 boys, whereas before they were the top 22 students, which usually favored the boys because the girls would decline to attempt to test in so that the boys could take the slots. The Anglican Church of Melanesia is very gender divided, in fact women and men are required to sit on opposite sides of the church for all 13 hours of it per week, so my work in this areas was subtle and small. Besides using my own privilege as a white man to set an example, there was very little to be done without crossing in to the territory of preaching that my culture is correct and they must change. The subtle

\footnotetext{
${ }^{37}$ Taf Tumas translates to: tough too much. It means someone is strong, powerful, successful, etc.
} 
difference here is that by setting an example, they can simply see the effect and choose to follow if they agree as opposed to pushing my own ideals on to their behaviors. More than a few male teachers never followed my laundry or cooking examples, but many of my male students noticed. This, above all else, is the story that the Peace Corps hears from heads of state to CEOs 50 years later about what inspired them to go to college or get involved in their community.

Outside the classroom my work on the GAD committee helped implement two full-blown workshops with 80 and 130 page manuals (in Bislama) to match. They were the summer-camp-like Kamp GLOW/BILD (Girls Leading Our World / Boys In Leadership Development, or $\mathrm{KGB}^{38}$ for short) and Gender Based Violence Prevention (GBVP) which operated more like a retreat style workshop. Both were a Training Of Trainers (TOT) type event, which was how we made the programs sustainable. While the GBVP TOT program taught me a great deal in how to talk about sensitive subjects and how adult learners in Vanuatu think and work, the KGB TOT program was far more useful in my work as a teacher. Particularly because the GAD committee hosted the annual KGB TOT at SPC during my second year of service. As a result, in addition to the many Peace Corps volunteers and their counterparts in attendance, 25 of SPC's students were able to attend as well. At the workshop they learned about many of the things that were not appropriate in or related to class, like sex ed and managing relationships. I learned so many lessons about what does and does not work for teaching these topics and

\footnotetext{
${ }^{38}$ In Bislama, Camp = Kamp, and KGB has no relation whatsoever to the Komitet gosudarstvennoy bezopasnosti, the Soviet Union's Committee for State Security
} 
it directly helped my ability to integrate and have hard or sensitive conversations in Bislama.

\section{Kamp GLOW/BILD}

The first and most important part of the KGB program requires that the methods used for learning are participatory. The techniques I learned here (discussed above) were instrumental in helping me develop effective curriculum for my classroom. The second part of the program requires that a relationship is built with the program's participants over the course of the week, because in such a low IDV culture, students will not learn from you if you do not have (very personal by American standards) relationships with them. This is only true for sensitive topics like Sikmun (Menstruation) and Kastom. More specifically, the GAD Vanuatu committee has an exercise called Leadership Compass that is derived from a Native American tool. It allows people to better understand their own working and communication styles. I was able to use this tool with both the staff and students at SPC to help improve working relationships and the overall learning environment. By understanding more how each person thinks, works, and communicates, many staff benefited tremendously. It is also important to remember that staff at a senior secondary school like SPC might change out (from transfers) as often as every 1 to 2 years. I know that I gained enormous benefit from learning that over $60 \%$ of my school's staff were South on the compass, which corresponds to the emotionally aware healer. In stark contrast, I am primarily a North as a take charge, get it done, warrior. Yet another tool I was able to use with my students was called Making Decisions, which taught them the consequences of their actions as well as the long term implications. It works via a life-simulating game in which students make decisions, like school vs work, or play vs 
study, or married vs single, or saving vs spending. Not only can students get into loops (just like in life) but they can also cut themselves off from certain sections by making irreversible decisions. At the end of the game, they end up in one of 13 categories that separate them by likely job, income level, where they live, how close they are to family, etc. I as an American might assume that more money and a better job is better, however one of the lessons that GAD taught me is Vanuatu's low MAS. Such forms of individual accomplishment mean little in Vanuatu culture. As always, the primary strength of these tools is in that they are participatory and in Bislama.

\section{Gender Based Violence Prevention}

My work in developing the GBVP TOT program also allowed me to better understand the methods by which adult learners think and work. Surprisingly, what I viewed to be trivial and patronizing exercises for adults were quite well received. I presume this is due to the low education level of those participating. For instance, an exercise where skits are required to act out conflict resolution scenes seemed very childish and patronizing to me, but the adults of the workshop loved it and it generated great discussion. I brought this technique that I would have never thought to try back to the staff at my school and the same effect happened. Another thing that I discovered, was that with adult learners, using a goal-oriented approach that makes it clear what the goals are is critical. Nebulous, poorly defined, absent, or even just non-applicable goals do not work with adult learners. Upon recognizing this I incorporated very clear and defined goals into my work with the staff of SPC. For instance, with my counterpart I started expressing (more often and more specifically) the goals behind each and every technique I used for both his training and our co-teaching. Immediately, I began to notice a 
difference where he and the other staff at SPC seemed slightly more enthusiastic about the work, presumably due to their newfound understanding of its purpose.

Whether it was role models, communication styles, adult learning methods, understanding hormones that control teenagers or any of a hundred other concepts, my work on the GAD committee was instrumental in my effectiveness as a teacher, as a volunteer, and as a staff member. I could not recommend GAD work more highly for future volunteers and hope that more concepts like this will penetrate the world of formal education in Vanuatu. As St. Patrick's College's motto says: "Educating the Whole Person" 


\section{Chapter VIII: Analysis}

\section{Limitations of Methodology}

The nature of Peace Corps service is that it is experiential by nature, and thus my observations are anecdotal. While they might well provide qualitative information, it is important to remember that this information was not obtained through formal research methods. As such, the opinions expressed throughout this report cannot be taken as researched and verified facts and must be assumed to contain a certain degree of both bias as well as the observer effect. They are the opinions of a single Peace Corps volunteer from a single school in Vanuatu. These opinions cannot in good faith be said to represent as accurately the same conclusions across all schools in all of Vanuatu. They are also not the opinions of the Peace Corps, Michigan Tech, St. Patrick's College, the Vanuatu Government, or any departments or individuals within these entities.

Some additional limitations of this work include the unreliable nature of data and information in Vanuatu. Often facts and figures are not completely correct, not because of poor research methods but because there does not exist a standard authority for correct information in many areas. For instance, the number of secondary schools that teach through year 13 changes every year and this number depends not only on when it is measured but if the measurement is based on current student enrollment and teaching or the previous year's exam results. Many examples of such information that Americans would never consider could have much variance or bias, often do in Vanuatu.

Finally with regard to limitations, it is important to note that Hofstede's Cultural Dimensions are not perfect. They have received criticisms over the years, most notably 
McSweeney [14] and Ailon [13], both of which Hofstede responded to. Finally, it is extremely important to note that Hofstede's Cultural Dimensions are relative quantitative measurements of national cultures, not individuals. While individuals often do fall within a small variance plus or minus their own culture's value for each HCD, individuals are by definition individual. They can be drastically different from their own culture's HCD values.

\section{Future work at SPC}

St. Patrick's College is a thriving school in Vanuatu, but IT/CS education is no easy task in the developing world. Future work at SPC should revolve around sustainably implementing curriculum and teacher training. Unfortunately, it must be noted that this work will not be undertaken by a Peace Corps volunteer. The Anglican Church of Melanesia decided (toward the end of my service) that the school's goals and the goals of the Peace Corps are not as compatible as they once were. In my opinion this is primarily a political decision. The US Peace Corps had spent over 4 years investing time and training into my counterpart, Newman Tahi, and he was replaced by the friend of an administrator without cause. In addition, the Peace Corps asked for a reason for the end of this relationship and none was given. Regardless, it would not matter. Peace Corps Vanuatu has also ended the entire Vanuatu IT project as a result of inability to support the project with qualified staff to manage the program.

Programs that have been successful at teaching Computer Science might be introduced in Vanuatu, like Hour of Code events and the Hopscotch app for iOS. In fact, an Hour of Code event took place at Port Vila International School, which offers quality 
education through year 10. While this school is exclusively for the children of affluent expatriates, it is only a matter of time before these events start bleeding out into Vanuatu's public schools and years 11-13. This will most likely occur once internet can be reliably used in an educational environment outside of the capital. Peace Corps is also organizing an ICT camp for girls to take place in the capital city in May 2016 [17].

\section{Related Work in the United States}

A search for related literature on the subject of IT/CS education in Vanuatu, Melanesia, and the developing world as a whole returned very little work. What did exist seems to be primarily the work of institutions attempting to assess the current state of affairs within their own institution and this was usually at the post secondary level. There is an absolute wealth of research on the topic of K12 CS education in the developed world, yet virtually no work of the same type in the developing world. This could be a very interesting avenue to explore in future works. Most notably: why?

The lack of literature regarding CS education in the developing world not withstanding, there are some aspects of this experience that might be applicable. For instance, anecdotes of how to better serve international undergraduate students in the US who come from similar cultures and socioeconomic backgrounds. As a graduate teaching assistant myself, I can imagine applications where we might attempt to make courses more diverse in their approaches for students who have HCD values opposite that of the United States, particularly Individualism and Power Distance. For example, individual presentations to a classroom of students on a research topic requires a very high power 
distance. The same learning goals might be more easily achieved for low PDI students with an online discussion forum or group presentations.

\section{Cultural Challenges and New Approaches}

Information Technology and Computer Science education in Vanuatu is an interesting endeavor, albeit a difficult one at the best of times. Through my experiences in the Peace Corps, teaching at St. Patrick's College in Vanuatu, I discovered, learned about, analyzed, sometimes succeeded at solving, and sometimes failed at solving some of the many challenges facing IT/CS education there. In my experience, these challenges fall under one of two very broad categories. The first being cultural and the second being logistical. At first glance, many people think that this order should be reversed, that the logistical challenges are more important and more difficult to resolve. Nothing could be further from the truth. Frankly, the intercultural differences during my teaching experience (as an American) in Vanuatu presented far greater challenges to teaching, training, and capacity building of the individuals and the institutions they serve. Regardless, new approaches were attempted for both overarching types of challenge, and some general trends were discovered about what new approaches might need to look like to be successful and more importantly, sustainable.

Most broadly, cultural difference based challenges almost always required some degree of compromise on the part of (usually) the teacher and (sometimes) that of the students/staff. Whereas in the United States assignments might have a strict due date, this approach does not work in Vanuatu. My experience indicates that a more relaxed approach to time in general was not only more successful but was also less stressful for 
both teachers and students. It was a difficult (at best) thing to do, but absolutely necessary for the success of IT/CS education at SPC. A relaxed approach to time, whether in lesson planning or course structuring or assignment deadlines, allowed for a less adversarial classroom environment and a more productive student mentality. It provided for a measure of compromise in which the educational method met the student's culture half way. In adopting just a bit of island time, this cultural divide was bridged just enough to promote student success more so than either approach individually ever could have.

Perhaps one of the largest cultural differences in Vanuatu was that of Gender Roles. This too required compromise on both my part and on the parts of the staff and students at SPC that I worked with. By not attempting to change their gender roles I gained their respect and was placed in a position in which I could serve as a role model. This was especially true because many international aid organizations that send volunteers did so with little to no cultural training. As Peace Corps preaches, my integration was my salvation. When I washed my own clothes, it was noticed. When I cooked for myself, it was noticed. When I insisted that my new Computer Studies students be precisely half female and half male, it was noticed. So too was it noticed when I sat with only the men in church and drank Kava with only the men at night. Embodying this compromise and choosing which gender roles were most critical gained me respect with many, most particularly my female students. Being younger and more open to Western ideals, this compromise allowed many more of my students to form quality relationships with me as a teacher. Had I not embraced such an ideal within my own behavior, my choice would have been to alienate the majority of one group or another. 
Also of the compromising style was respecting power distance. The trouble with high power distance as a Peace Corps volunteer was that my purpose in being there was to provide development assistance and participate in intercultural exchange. Being a volunteer in a single school with zero official power, and thus at the bottom of any conceivable power ladder, means that a certain degree of fluctuation was sometimes needed. The key was in respect. Not in the sense of professionalism as one might view respect in the Western world, but in the sense of reverence. It took the leadership concept of leading from behind to an entirely new level and it was not an easy thing to do. It required an approach that was entirely foreign, where the respect of leaders was first gained through social situations over long periods of time. Then and only then might suggestions, comments, and even complaints be appropriate to make. It was inherently slower and less effective than open communication and confrontation, but in a culture like Vanuatu, violating power distance too much or too often backfired far worse.

Uncertainty is one of the only certainties in the developing world, and Vanuatu is no exception. Because teaching is a very methodical process that follows a somewhat inflexible list of steps, this could also be a very difficult cultural dimension to adapt to. The only way to truly let go of the control I (as a teacher) loved so much was to recognize that the culture of Vanuatu does not promote either individualism or accomplishment. Rather, it promotes a shorter term view of education and life in which the here, the now, and family are more important than achievement and personal gain. This priority difference meant that uncertainty was not a problem, it simply was, and trying to constantly plan for avoiding it was never successful, rather less sustainable. One is far better off simply becoming well practiced at reacting to the unexpected than 
attempting to plan for it. Indeed, beyond a Peace Corps or a Vanuatu lesson, this is truly a life lesson.

The only cultural dimension that required a true identity shift on the part of the students at SPC was that of collectivism. Some of the basic principles of education are those of individual thought, critical thinking, and personal knowledge/intelligence. These concepts, while not diametrically opposed to collective thought or a collectivist mindset, are however mutually exclusive from formal IT/CS education. It is also possible that this is true of education in general. Of course a certain degree of understanding and adaptation was still necessary when teaching, but promoting the idea of individualism (in so far as education was concerned) can be and always was a goal for me. I firmly believe that this improved student test scores, confidence, and overall academic success.

Language was a slightly different challenge. Not really a logistical challenge because language is of course a primary root of culture, but also not really a cultural challenge because the best approach I found was mostly logistical. The way in which language barriers were faced was via teaching adaptation to help students bridge the language gap on their own. Many senior secondary students were mostly familiar with English, but using simpler words and sentence structures helped them nonetheless. Most important was to simply break through the ethnocentrism of teaching in my native language so that I could constantly be aware of my language choices and their needs surrounding it. 


\section{Logistical Challenges and New Approaches}

The logistical challenges to CS/IT education in Vanuatu, at first glance, seemed to be insurmountable, and indeed they were extremely difficult. However, they paled in comparison to the cultural challenges. All the money in the world without the necessary cultural understanding to use it effectively moved projects nowhere very fast. Or even worse, backwards in creating a dependency or an expectation that then must be unlearned later on. These types of problems usually arise as a result of top-down development, particularly where the research and understanding of the underlying cultural roots is lacking. In this there are two schools of thought. The first is in avoiding such top-down created problems by implementing excruciatingly slow (and more often unsuccessful) projects without serious resources. For instance to fundraise for buying computers from locals who had very little disposable income. Even if successful, using the money correctly was still a major challenge. The second school of thought was to attempt to guide the project by teaching and training those whom I worked with about the process and planning as much as was possible. By writing the grant themselves and forming a committee with responsibilities and consequences, they created not only a sense of ownership but also gained a greater understanding of the project. Regardless of which school of thought one followed, the key to both was rooted in considering sustainability and cultural understanding from the ground up.

In addition to money and resources, two other factors were required for the success of logistics related approaches: patience and prior knowledge. Projects that solved logistical challenges like a lack of computers or electricity took time. A lot of time. The more difficult the project was and the more money that was to be spent always 
directly influenced the amount of time it took to complete. Other factors could also influence the project's timeline, like cyclones and land ownership disputes. Patience and expecting a very long timeline from the beginning was key to both success and happiness in such projects.

In the planning phase for such projects, prior knowledge was a critical component. No amount of patience or cultural understanding makes up for some of the information and experience that is simply necessary to solve logistical challenges. This prior knowledge usually came from me or subject matter experts and was necessary for SPC staff to learn how to complete, for example, a sustainability projection or a depreciation schedule. No amount of effort on the part of a local leader could teach them how to write a grant if they could not even type or use a computer. Other examples include things like fixing computers, setting up computer labs, installing solar power systems, and (arguably the most important for my service) was knowledge and experience with teaching mechanics. Without work and input from such motivated individuals from outside of the community, prior knowledge wasn't shared and it created consequences for the success of logistical approaches. 


\section{Chapter IX: Conclusion}

The Republic of Vanuatu is in an exciting time in its history. Having just declared independence from England and France a mere 36 years ago, they have made tremendous progress in building their nation with a beautiful fusion of culture and modern technology. In them is a spark for new technology to join the modern world, which has recently burst into flame due to the arrival of the first ever under-sea internet cable in January of 2014. In just a few years, it has driven the integration and education of technology at incredible speeds. If one thing is for certain it is that change surrounding IT/CS in Vanuatu will be growing exponentially for many years to come. As they make progress toward a self-sustaining IT/CS education infrastructure, the largest portion of that responsibility falls upon the current and future IT/CS educators throughout Vanuatu. Any project trying to bring computers and internet to places that barely have roads and running water is an endeavor to be proud of and is just as important for securing the future of Vanuatu's people, economy, and culture. In an age where computers and technology are ubiquitous, they must continually adapt the methods by which they preserve their culture while simultaneously joining the $21^{\text {st }}$ century. The fusion of their beautiful culture and modern technology is a goal shared by the Peace Corps and I can imagine no better organization for providing volunteers to help realize that goal. It is my hope that this report might one day serve to help prepare a Peace Corps volunteer for their assignment, wherever they may be placed. It is also my hope that this report has shed light on the beautiful culture, strive for independence, and true spirit of the people of the Republic of Vanuatu. 


\section{References}

1. Sir Clements Markham (ed.), The Voyages of Pedro Fernandez de Quiros, 1595 1606, Hakluyt Society, 1904, Vol.1, pp.251, 478; Brendan Whyte, “Australia or Austrialia? A Correction", The Globe, no.69, 2011, p.51; Rupert Gerritsen, "A Note on Australia or Austrialia",The Globe, no.72, 2013, pp.23-30; Margaret Cameron-Ash, "Juggling 'Australia', 'Austrialia' and 'New Holland'", The Globe, no.73, 2013, pp.29-38.

2. Hess, Sabine C. (July 2009). Person and Place: Ideas, Ideals and the Practice of Sociality on Vanua Lava, Vanuatu. Berghahn Books. p. 115. ISBN 978-1-84545599-6.

3. The Peace Corps Welcomes You to Vanuatu. Peace Corps (May 2007).

4. International Religious Freedom Report 2007: Vanuatu. United States Bureau of Democracy, Human Rights and Labor (September 14, 2007).

5. "Welcome to The Hofstede Centre." Cultural Insights. N.p., n.d. Web. 22 Mar. 2016.

6. "Census \& Surveys". Vanuatu National Statistics Office. Retrieved 27 May 2013.

7. "Vanuatu Consolidated Legislation - 2006." Constitution of the Republic of Vanuatu. N.p., n.d. Web. 22 Mar. 2016.

8. "Vanuatu Court Sentences 14 MPs to Jail for Corruption." BBC News. BBC, 22 Oct. 2015. Web. 22 Mar. 2016.

9. "Usage of Content Languages for Websites." Usage Statistics of Content Languages for Websites, March 2016. N.p., n.d. Web. 22 Mar. 2016.

10. "Nelson Mandela Quote." BrainyQuote. Xplore, n.d. Web. 22 Mar. 2016.

11. "A Quote by Albert Einstein." Goodreads. N.p., n.d. Web. 27 Mar. 2016.

12. "Q2 2015 State of the Internet - Security Report | Cloud Security Trends."State of the Internet. N.p., n.d. Web. 30 Mar. 2016

13. Ailon, G. (2008). Mirror, mirror on the wall: Culture's Consequences in a value test of its own design. The Academy of Management Review, October 2008, 33(4):885-904].

14. McSweeney, B. (2002), Hofstede's model of national cultural differences and their consequences: a triumph of faith - a failure of analysis. Human Relations, 55(1): 89-117.

15. "Participatory Methods." Impact Evaluation -. N.p., n.d. Web. 09 Apr. 2016.

16. "FET Free Timetabling Software :.:" FET. N.p., n.d. Web. 09 Apr. 2016.

17. http://www.smartsistas.vu/

18. "Maximum Advertised Speed Available." National Broadband Map. N.p., n.d. Web. 


\section{Appendix A: The History Of St. Patrick's College}

\section{BRIEF HISTORY OF ST. PATRICK'S COLLEGE}

St Patrick's college is an Anglican Church institution and is one of the oldest schools in the country. In the early Missionary days in the late 1800's, the Church of England took young men from Melanesia to New Zealand to train them to be missionaries. After only 30 years the school in New Zealand was relocated to Norfolk islands and was there for about the same time since the former country was too cold for the Melanesians. Even Norfolk Island was regarded too foreign for the young men so in the early 1900's the church of the provinces of New Zealand decided to establish the school in Melanesia itself.

In 1903 a primary school was built at Vureas Bay on Vanua Lava in the Banks group by an Irish clergy man, Fr. Mc Oferral and he named it St. Patrick's school, Vureas. St. Patrick's is Ireland's Patron Saint as Fr. Mc Oferral was Irish; it was pride and honour for him to name the school he built after his Patron saint.

The school remained at Vureas Bay from 1903 to 1935 when another decision came up to move the school to Nawalu valley on Ambae Island. There were many reasons for this relocation. There were numerous problems with malaria that caused many deaths among students and teachers. The land was not productive enough and it was too isolated from the main centre of activity. The school then moved to the present location with its well known name "Vureas" and is retained to this day.

St Patrick's college now is completely different from what it used to be. In the 1970's St. Patrick's college was upgraded to junior secondary school slowly phasing out the primary programme. The college began to develop further in early 1990's and eventually in 1996 it began teaching year 11 and 12 Pacific Senior Secondary Certificate (PSSC) programme and in 2001 the university of the south pacific Foundation programme was introduced for the first time as the year 13 programme. In 2004 the college phased out Foundation programme and took in South Pacific Form seven Certificate (SPFSC) which is still offered in the college.

Thank you,

Kathy Tahi

Academic Principle

St. Patrick's College 